\title{
Drag Prediction for the NASA CRM Wing-Body-Tail Using CFL3D and OVERFLOW on an Overset Mesh
}

\author{
Anthony J. Sclafani, ${ }^{*}$ Mark A. DeHaan, ${ }^{\dagger}$ John C. Vassberg ${ }^{\ddagger}$ \\ The Boeing Company, Boeing Research \& Technology, Huntington Beach, CA, 92647, USA \\ Christopher L. Rumsey ${ }^{\S}$ \\ NASA Langley Research Center, Hampton, VA, 23681, USA \\ Thomas H. Pulliam ${ }^{* *}$ \\ NASA Ames Research Center, Moffett Field, CA, 94035, USA
}

\begin{abstract}
In response to the fourth AIAA CFD Drag Prediction Workshop (DPW-IV), the NASA Common Research Model (CRM) wing-body and wing-body-tail configurations are analyzed using the Reynolds-averaged Navier-Stokes (RANS) flow solvers CFL3D and OVERFLOW. Two families of structured, overset grids are built for DPW-IV. Grid Family 1 (GF1) consists of a coarse (7.2 million), medium (16.9 million), fine (56.5 million), and extra-fine (189.4 million) mesh. Grid Family 2 (GF2) is an extension of the first and includes a superfine (714.2 million) and an ultra-fine (2.4 billion) mesh. The medium grid anchors both families with an established build process for accurate cruise drag prediction studies. This base mesh is coarsened and enhanced to form a set of parametrically equivalent grids that increase in size by a factor of roughly 3.4 from one level to the next denser level. Both CFL3D and OVERFLOW are run on GF1 using a consistent numerical approach. Additional OVERFLOW runs are made to study effects of differencing scheme and turbulence model on GF1 and to obtain results for GF2. All CFD results are post-processed using Richardson extrapolation, and approximate grid-converged values of drag are compared. The medium grid is also used to compute a trimmed drag polar for both codes.
\end{abstract}

\section{Nomenclature}

$\begin{array}{ll}A R & =\text { wing aspect ratio, } b^{2} / S_{\text {ref }} \\ C F D & =\text { computational fluid dynamics } \\ C_{D} & =\text { drag coefficient, } D r a g /\left(q_{\infty} S_{\text {ref }}\right) \\ C_{L} & =\text { lift coefficient, } \operatorname{Lift} /\left(q_{\infty} S_{\text {ref }}\right) \\ C_{M} & =\text { pitching moment coefficient, Moment } /\left(q_{\infty} S_{r e f} C_{\text {ref }}\right) \\ C_{P} & =\text { pressure coefficient, }\left(P-P_{\infty}\right) / q_{\infty} \\ C_{r e f} & =\text { wing reference chord } \\ C & =\text { chord } \\ \text { count } & =\text { drag coefficient unit, } 0.0001 \\ D P W & =\text { drag prediction workshop } \\ F N S & =\text { full Navier-Stokes } \\ i_{H} & =\text { horizontal tail incidence } \\ R A N S & =\text { Reynolds-averaged Navier-Stokes } \\ S_{\text {ref }} & =\text { wing reference area } \\ T L N S & =\text { thin-layer Navier Stokes }\end{array}$

\footnotetext{
* Aerodynamics Engineer 4, AIAA Senior Member.

† Aerodynamics Engineer 5, AIAA Senior Member.

*Aerodynamics Engineer 6, Technical Fellow, AIAA Fellow.

${ }^{\S}$ Senior Research Scientist, AIAA Associate Fellow.

${ }^{* *}$ Research Scientist, AIAA Associate Fellow.
} 


\section{Introduction}

$\mathrm{T}$ HE Drag Prediction Workshop (DPW) has provided users and developers of Computational Fluid Dynamics (CFD) programs an opportunity to assess how well current methods compute aerodynamic characteristics of modern transport aircraft in various high speed configurations. The underlying theme of DPW is accurate cruise drag prediction which has become a requirement for Reynolds-averaged Navier-Stokes (RANS) flow solvers employed within a production aircraft design environment (see References 1 and 2). However, workshop objectives go well beyond computed forces and moments. Items discussed in past workshops include grid generation requirements for convergence studies, turbulence modeling with flow separation, and general trends in structured vs. unstructured results.

To date, four Drag Prediction Workshops have been conducted. There are a number of publications that provide background information on the first three workshops, such as References 3 and 4, so this discussion will be limited to an overview of the fourth workshop. DPW-IV represents a break in the DPW series because it utilized a newly designed wind tunnel model called the NASA Common Research Model (CRM) which was developed specifically for CFD validation studies. ${ }^{5}$ The CRM, shown in Figure 1, is an ideal test case geometry for DPW-IV for many reasons.

- Designed with CFD validation as a high priority - minimal flow separation

- Relevant geometry with varying degrees of complexity (wing-body, wing-body-tail, wingbody-tail-pylon-nacelle)

- Publicly available geometry, grids, CFD results, and test data

- Newly acquired, high quality test data from multiple facilities

- Experimental data not available until after the workshop

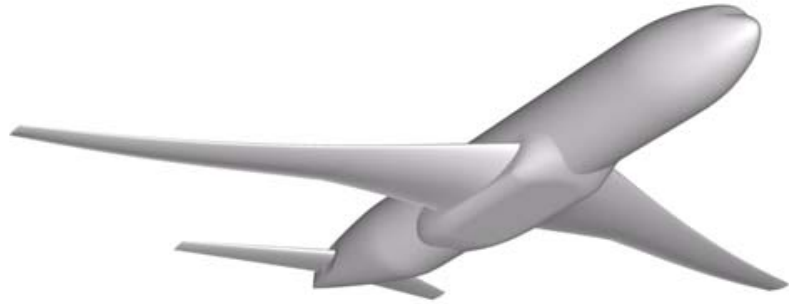

Figure 1. CRM Wing-Body-Tail Configuration.

A wing-body-tail configuration was included in DPW-IV allowing for prediction of trimmed cruise drag which has not been studied in past workshops. CRM test data did not exist at the start of DPW-IV making it a true blind workshop. Afterward, two wind tunnel tests were conducted but data remained publicly unavailable when this paper

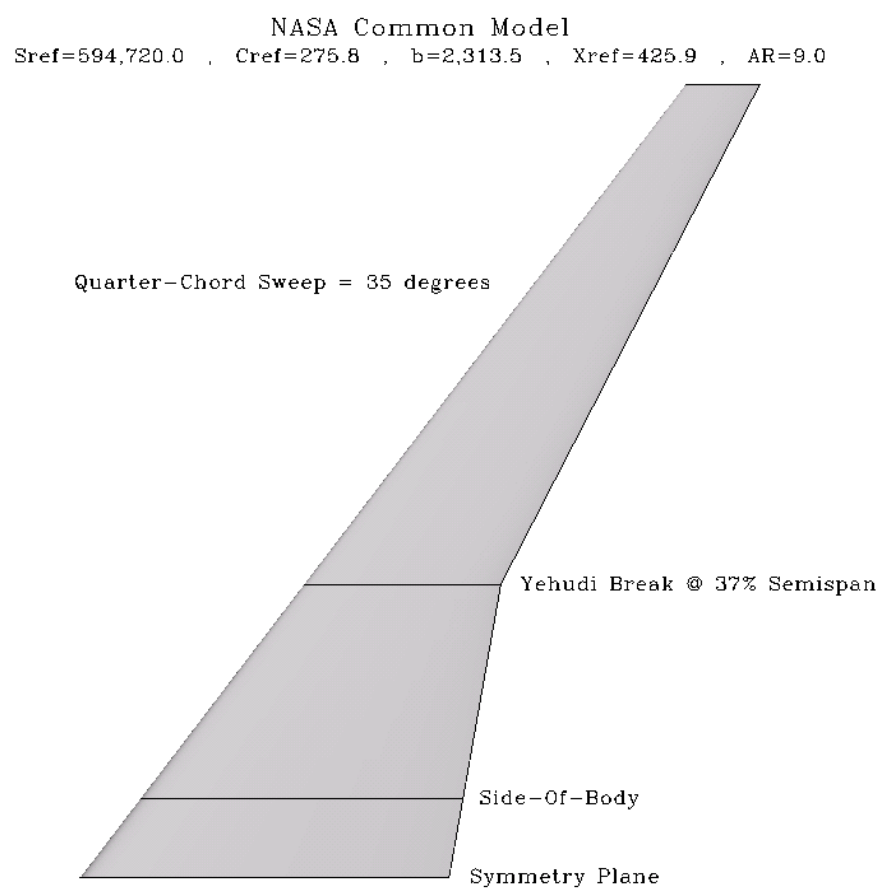
was written. The focus here will be on CFD-toCFD comparisons.

The CRM experimental data is being released by Rivers ${ }^{6}$. The DPW-IV summary paper ${ }^{7}$ includes some of this test data for a first look at how CFD results submitted to the workshop compare to the wind tunnel.

This paper summarizes the NASA CRM overset grid results from two RANS flow solvers: CFL3D and OVERFLOW. DPW-IV consisted of three test cases but only the first was required. Both codes were run on the set of grids provided to all workshop participants for Test Case 1 which is a combined grid convergence and downwash study. While the focus of this paper will be on a code comparison for the two studies of Test Case 1, additional OVERFLOW results will be discussed. These additional results are from the workshop's optional test cases and from extended grid resolution studies.

Figure 2. CRM Wing Planform. 


\section{Geometry Description}

The CRM represents a modern, transonic, transport configuration with a horizontal tail, nacelle, and pylon. To simplify the test case for CFD validation purposes, the nacelle and pylon were not included in the geometry for

\begin{tabular}{ll|}
$S_{\text {ref }}$ & $594,720.0 \mathrm{in}^{2}$ \\
$C_{\text {ref }}$ & $275.80 \mathrm{in}$ \\
$S_{p a n}(b)$ & $2,313.50 \mathrm{in}$ \\
$X_{\text {ref }}$ & $1,325.90 \mathrm{in}$ \\
$Y_{\text {ref }}$ & $468.75 \mathrm{in}$ \\
$Z_{\text {ref }}$ & $177.95 \mathrm{in}$ \\
$\lambda$ & 0.275 \\
$\Lambda_{\mathrm{C} / 4}$ & $35^{\circ}$ \\
$A R$ & 9.0 \\
\hline
\end{tabular}

Table 1. CRM Reference Quantities. DPW-IV. Figure 1 shows the CRM wing-body-tail configuration. Note the inclusion of a wing-body fairing and scrubbing seal for the horizontal tail.

The wing design is optimized for a cruise Mach of 0.85 and lift coefficient of 0.5 with the wing planform shown in Figure 2. Some of the CRM wing reference quantities are given in Figure 2 but a more complete list is provided in Table 1.

Three horizontal tail settings $\left(\mathrm{i}_{\mathrm{H}}\right)$ were provided to workshop participants. This made a total of four configurations to be analyzed: tail-off, $i_{H}=-2^{\circ}, 0^{\circ}$, $+2^{\circ}$. More information on the CRM design requirements, optimization tools utilized, and overall geometry can be found in Reference 5 .

\section{Overset Grid Generation}

The DPW-IV organizing committee provided participants with a detailed set of guidelines to follow for the grid generation process. ${ }^{8}$ These guidelines include recommendations for wall spacing, stretching ratio, and grid size. The CRM overset grids were constructed using the DPW-IV guidelines as well as the current state of best practices for overset grid generation when accurate cruise drag prediction is required. ${ }^{2,9}$ For the grid convergence study, a process has been established for creating a consistent set of grids. The first step of this process is building the medium grid.

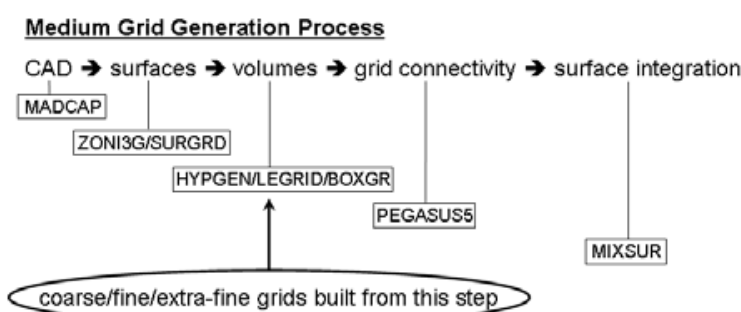

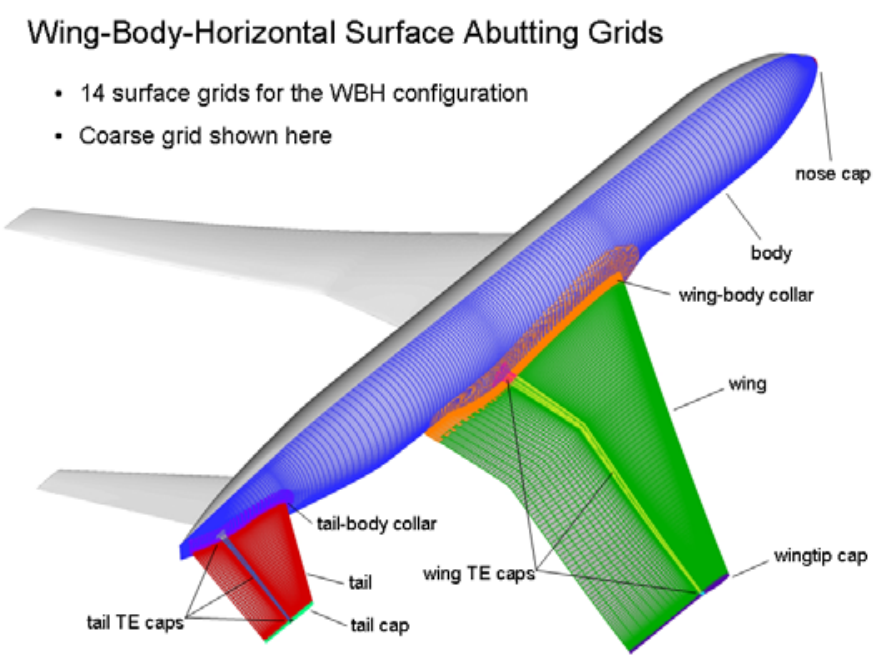

Figure 4. CRM Overset Surface Grid Layout.

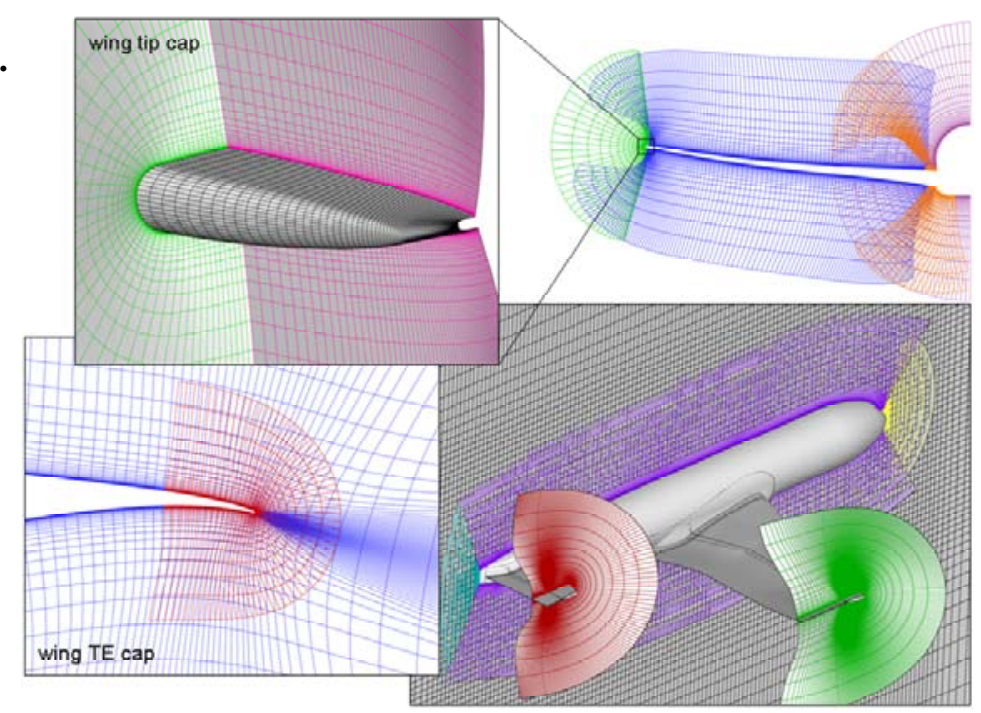

Figure 5. CRM Overset Volume Grids.

Figure 3. Overset Grid Generation Process.

\section{A. Medium Grid}

The medium grid was built using tools and techniques suitable for drag-quality design studies. The steps followed to take the CRM geometry through the grid generation process are illustrated in Figure 3. The geometry was provided in the form of Initial Graphics Exchange Specification (IGES) files, so the first step was to extract points from the surface definition. This was done using a Boeing-developed program called MADCAP which can import geometry from different file types (e.g. IGES) for use in structured and unstructured grid generation. Once the geometry was imported into MADCAP, an initial set of well defined surface grids were 
created and exported to another Boeing tool called ZONI3G. ${ }^{10}$ This interactive graphical program was used to finalize surface point distributions, add wakes to lifting surfaces, generate cap grids and manipulate surfaces for optimum grid overlap. Unlike MADCAP, ZONI3G does not work directly on CAD geometry so the dense network of points exported from MADCAP was used as a CRM loft representation. Final surface grids built using ZONI3G were projected back to the loft representation when needed. The fuselage-side of the wing and tail collar grids were the only surfaces generated using the Chimera Grid Tools (CGT) package. ${ }^{11}$ A CGT program called SURGRD was utilized for this purpose. The surface grid layout for the CRM wingbody-tail configuration is comprised of 14 surface abutting regions and is shown in Figure 4.

Volume grid generation was the next step for the medium mesh (see Figure 3). The volume grids were built using a script system $^{12}$ developed at NASA which is intended to work with the CGT package. This script system brings a degree of automation to the overall process by defining boundary conditions for each grid, organizing components with a master configuration file, and driving the CGT programs with a master input file. A script tool called BuildVol generates volume grids where surface grids are run through one of two hyperbolic grid generators (HYPGEN $^{13}$ and LEGRID) and cartesian box grids are created using BOXGR. Different views of the volume grids are shown in Figure 5. The image in the lower right portion of Figure 5 includes the symmetry plane from a mid-field box grid.

A program called PEGASUS5 ${ }^{14}$ was used to establish communication between the individual volume grids. This is a step in the process known as domain or grid connectivity. The grids are connected by cutting holes where points fall inside geometry or where another grid is found to have better spacing, and then defining interpolation stencils at grid and hole boundaries. After PEGASUS5 was run, a force and moment integration surface was created using another NASA program called MIXSUR. This program eliminates grid overlap on the surface and connects

Body Grids
\begin{tabular}{|l|l|l|l|l|}
\hline grid & $\mathrm{J}$ & $\mathrm{K}$ & $\mathrm{L}$ & total \\
\hline coarse & 217 & 73 & 79 & 776,209 \\
& $75 \times \mathrm{m}$ & $75 \times \mathrm{m}$ & $7.75 \times \mathrm{m}$ & $7.43 \times \mathrm{m}$ \\
medium & 289 & $\mathbf{9 7}$ & $\mathbf{6 5}$ & $\mathbf{1 , 8 2 2 , 1 4 5}$ \\
& 433 & 1.45 & 97 & $6,090,145$ \\
fine & $1.50 \times \mathrm{m}$ & $1.49 \times \mathrm{m}$ & $1.49 \times \mathrm{m}$ & $3.34 \times \mathrm{m}$ \\
& 649 & 217 & 145 & $20,420,785$ \\
extra-fine & $1.50 \times \mathrm{xf}$ & $1.50 \times \mathrm{ff}$ & $1.49 \times \mathrm{xf}$ & $3.35 \times \mathrm{m}$ \\
\hline
\end{tabular}

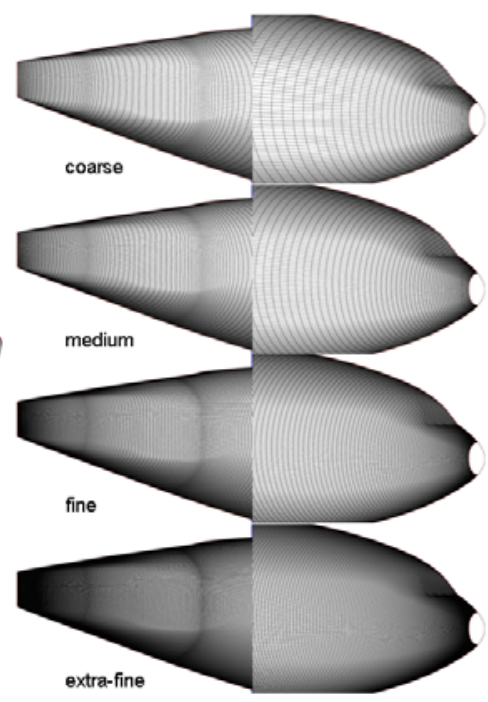

Figure 6. CRM Overset Body Grid Family.

Wing-Body Collar Grids
\begin{tabular}{|l|l|l|l|l|}
\hline grid & $\mathrm{J}$ & $\mathrm{K}$ & $\mathrm{L}$ & total \\
\hline coarse & 289 & 37 & 49 & 523,957 \\
& $75 \times \mathrm{m}$ & $.75 \times \mathrm{m}$ & $75 \times \mathrm{m}$ & $43 \times \mathrm{m}$ \\
medium & $\mathbf{3 8 5}$ & $\mathbf{4 9}$ & $\mathbf{6 5}$ & $\mathbf{1 , 2 2 6 , 2 2 5}$ \\
& $\mathbf{5 7 7}$ & 73 & $\mathbf{9 7}$ & $4,085,737$ \\
fine & $1.50 \times \mathrm{m}$ & $1.49 \times \mathrm{m}$ & $1.49 \times \mathrm{m}$ & $3,33 \times \mathrm{m}$ \\
& 865 & 109 & 145 & $13,671,325$ \\
extra-fine & $1.50 \times \mathrm{f}$ & $1.49 \times \mathrm{f}$ & $1.49 \times \mathrm{f}$ & $3.35 \times \mathrm{m}$ \\
\hline
\end{tabular}
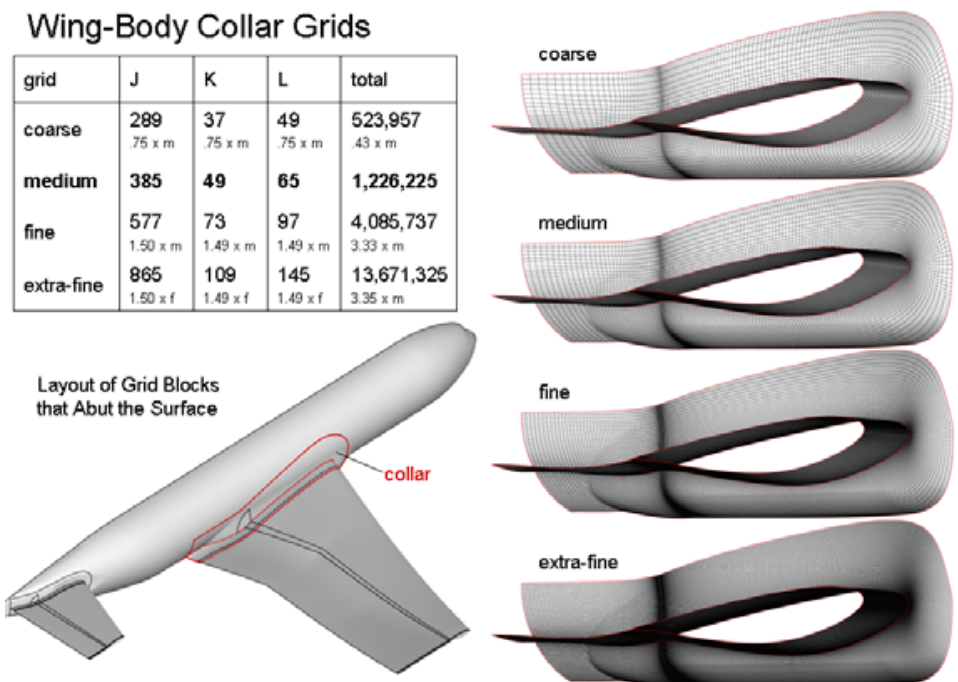

Figure 7. CRM Overset Wing-Body Collar Grid Family.

Wing Trailing-Edge Cap Grids
\begin{tabular}{|l|l|l|l|l|}
\hline grid & $\mathrm{J}$ & $\mathrm{K}$ & $\mathrm{L}$ & total \\
\hline coarse & 73 & 37 & 37 & 99,937 \\
medium & $75 \times \mathrm{m}$ & $75 \times \mathrm{m}$ & $.75 \times \mathrm{m}$ & $43 \times \mathrm{m}$ \\
& 97 & 49 & 49 & 232,897 \\
fine & 145 & 73 & 73 & 772,705 \\
& $1.49 \times \mathrm{m}$ & $1.49 \times \mathrm{m}$ & $1.49 \times \mathrm{m}$ & $3.32 \times \mathrm{m}$ \\
extra-fine & 217 & 109 & 109 & $2.578,177$ \\
\hline
\end{tabular}
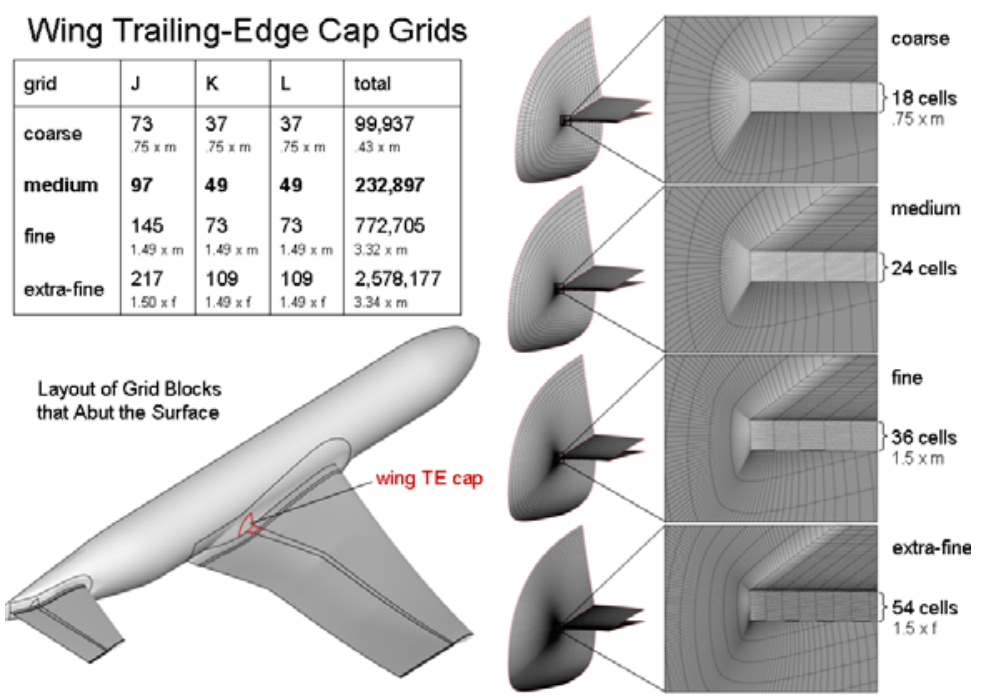

Figure 8. CRM Overset Wing Trailing Edge Cap Grid Family. 
Table 2a. Summary of Grid Parameters for the Wing-Body

\begin{tabular}{|c|c|c|c|c|c|c|}
\hline Grid & Points & $1 / \mathrm{N}^{2 / 3} \times 10^{5}$ & $1^{\text {st }}$ Cell Size & $\mathrm{y}^{+}$ & $\begin{array}{c}\text { Constant } \\
\text { Cells }\end{array}$ & $\begin{array}{c}\text { Growth } \\
\text { Rate }\end{array}$ \\
\hline Medium & $12,267,995$ & 1.88 & .00079 in & .66 & 3 & 1.19 \\
\hline
\end{tabular}

Table 2b. Summary of Grid Parameters for the Wing-Body-Tail.

\begin{tabular}{|c|c|c|c|c|c|c|}
\hline Grid & Points & $1 / \mathrm{N}^{2 / 3} \times 10^{5}$ & $1^{\text {st }}$ Cell Size & $\mathrm{y}^{+}$ & $\begin{array}{c}\text { Constant } \\
\text { Cells }\end{array}$ & $\begin{array}{c}\text { Growth } \\
\text { Rate }\end{array}$ \\
\hline Coarse & $7,221,233$ & 2.68 & .00104 in & .87 & 2 & 1.26 \\
Medium & $16,932,913$ & 1.52 & .00079 in & .66 & 3 & 1.19 \\
Fine & $56,531,489$ & 0.68 & .00052 in & .44 & 4 & 1.12 \\
Extra Fine & $189,413,153$ & 0.30 & .00035 in & .29 & 6 & 1.08 \\
\hline
\end{tabular}

Table 2. Summary of GF1 Overset Grid Parameters.

neighboring zones with zipper grids comprised of triangles. MIXSUR produces a closed integration surface used to compute forces and moments.

\section{B. Coarse, Fine, and Extra-Fine Grids}

As discussed in Reference 15, one requirement for a meaningful grid convergence study is uniform grid refinement. This means the grid family must be parametrically equivalent where a coarser mesh is a subset of the next denser mesh. For this reason, the coarse/fine/extra-fine grids were constructed using a different process than the one summarized in the previous subsection. The volume grids from the medium mesh are used as a starting point for all other grids in the family (see Figure 3 ). A purposebuilt program, developed at Boeing, redistributes points in the medium grid using a weighted-average cubic interpolation routine. The user has control over how many grid points are added or taken away from the input mesh and whether surface breaks are preserved. The end result is a family of grids where each index increases by a factor of 1.5 as prescribed in the DPW-IV Gridding Guidelines. ${ }^{8}$ Figure 6 includes a table of grid dimensions and images for the fuselage family of grids. The same information is given for the wing-body collar grid in Figure 7 and wing trailing-edge cap grid in Figure 8. The grid images in these two figures show surface breaks at the wing-body intersection and wing trailing-edge. The process used to build the grid family maintains these and all other surface breaks through at least three multi-grid levels.

The fuselage grid, wing-body collar grid, and wing trailing-edge cap grid summarized in Figures 6-8 are 3 of the 14 surface abutting grids that make-up the wing-body-tail configuration. With 2 mid-field box grids and 1 far-field box grid, this configuration has a total of 17 grids. The wing-body configuration is made-up of 11 grids. Table 2 summarizes some key grid parameters for both configurations as defined in the DPW-IV Gridding Guidelines.

\section{CFL3D Grid Requirements}

For the grid connectivity step shown in Figure 3, PEGASUS5 establishes boundary information (i.e. interpolants) at grid points or nodes. This is a requirement driven by the overset grid flow solver, OVERFLOW, which is a node-based code. CFL3D is a cell-center code, so a method was developed by the NASA LaRC author that forces PEGASUS5 to find overset grid interpolants at cell centers. This procedure involves utilizing the dual LaRC grid so nodes are located at cell centers. PEGASUS5 is then run on the dual grid. For more details, see Reference 14.

\section{Flow Solver and Computing Platform}

CFL3D ${ }^{16,17}$ and OVERFLOW ${ }^{18}$ are widely used RANS codes considered reliable and accurate for analyzing modern transport configurations, like the CRM, at or near the cruise design condition. This paper summarizes results from two studies where these flow solvers are run on a common set of overset grids. The first study focuses on a code-to-code data comparison utilizing the overset grids (GF1) built for DPW-IV. Some general information on how CFL3D and OVERFLOW were run for this study is given here. The second study is based on OVERFLOW analyses using grids (GF2) built outside the scope of DPW-IV. Additional flow solver and computing platform information for this second study will be discussed in Section V (Results).

\section{A. CFL3D}

CFL3D was developed at the NASA Langley Research Center as a cell-based RANS code for structured grids. It is considered a general purpose flow solver that can be used for both steady and unsteady simulations. The boundary layer is computed as laminar or turbulent with a choice of several different turbulence models. For DPW-IV, CFL3D was run in a steady-state, fully turbulent mode using the Spalart-Allmaras (SA) ${ }^{19}$ one-equation turbulence model and upwind Roe differencing. Viscous terms were computed in the wall-normal direction only, so the CFL3D dataset 
will be referred to as Thin Layer Navier-Stokes (TLNS). This information is provided in Table 3 along with the dataset name used to compare results. See Dataset 1 in Table 3.

CFL3D version 6 was run in parallel using 64-bit precision on several different computer clusters. For example, the fine grid (56 million points) was run on Columbia, which is a 10,240 CPU SGI Altix supercluster at NAS.

CFL3D can only be split up to run in parallel on as many processors as there are zones (17 in this case). No attempt was made in this study to improve efficiency by splitting the zones into smaller component pieces with better load balancing. Also, the code cannot perform mesh sequencing on overset cases; it is much more efficient to run on multiple-zone topologies consisting solely of 1-to-1 interfaces. To run 5000 iterations on the fine overset grid required approximately 300 hours of wall-clock time.

\section{B. OVERFLOW}

OVERFLOW was developed by NASA with numerous contributions from academia and industry. It is a nodebased RANS code specifically designed for structured overset grids. As with CFL3D, many options are available to the user such as 2D/3D, steady/unsteady, TLNS vs. full Navier-Stokes (FNS), and multiple turbulence models. Initially, both the Baldwin-Barth (BB) and SA turbulence models were used to analyze the CRM. However, the BB results showed excessive shock-induced separation on the mid-wing region when the grid was refined so only the SA data was submitted to DPW-IV. After the workshop, additional OVERFLOW runs were made using Menter's two-equation Shear Stress Transport $(\mathrm{SST})^{20}$ turbulence model. In addition to the turbulence model study, OVERFLOW was run with various combinations of differencing scheme and direction of viscous calculation. Table 3 summarizes these combinations for both codes. Datasets 1, 3, and 4 were completed prior to DPW-IV whereas Datasets 2 and 5 were part of follow-on studies.

OVERFLOW version 2.1t was run using 64-bit precision and grid sequencing to accelerate convergence. The Message Passing Interface (MPI) version of the code was run in parallel using a Linux PC cluster consisting of 1968 cores on 578 nodes. A wing-body-tail medium grid (16.9 million points) TLNS solution required 3.5 hours of wallclock time to complete 1000 iterations on 16 processors. For the design condition, full convergence was achieved after 4000 fine-grid iterations or 14 hours.

\begin{tabular}{|c|c|c|c|c|c|}
\hline Dataset \# & Dataset Name & Code & $\begin{array}{c}\text { Differencing } \\
\text { Scheme }\end{array}$ & $\begin{array}{c}\text { Turbulence } \\
\text { Model }\end{array}$ & $\begin{array}{c}\text { Thin Layer } \\
\text { or Full N-S }\end{array}$ \\
\hline 1 & C-SA-UPW-TL & CFL3D & Roe Upwind & SA & TLNS \\
2 & O-SA-UPW-TL & OVERFLOW & Roe Upwind & SA & TLNS \\
3 & O-SA-CEN-TL & OVERFLOW & Central & SA & TLNS \\
4 & O-SA-CEN-F & OVERFLOW & Central & SA & FNS \\
5 & O-SST-CEN-TL & OVERFLOW & Central & SST & TLNS \\
\hline
\end{tabular}

Table 3. Dataset Information for the Overset Grid Solutions.

\section{Results}

Participants were required to submit data on the first of three test cases defined for DPW-IV. The required case consisted of a grid convergence study and a downwash study. The other two optional test cases were a Mach sweep study and a Reynolds number study. Table 4 provides details on all DPW-IV test cases as well as a cross-reference to the datasets listed in Table 3.

\begin{tabular}{|c|c|c|c|c|c|c|c|c|c|}
\hline $\begin{array}{l}\text { Test } \\
\text { Case }\end{array}$ & Study Name & Mach & $\begin{array}{l}\mathrm{RN} \\
\text { (mil) }\end{array}$ & $\begin{array}{l}\text { Temp } \\
\left({ }^{\circ} \mathrm{F}\right)\end{array}$ & $\mathrm{C}_{\mathrm{L}}$ & $\begin{array}{c}\text { Alpha } \\
\text { (deg) }\end{array}$ & $\begin{array}{l}\text { Tail } i_{H} \\
(d e g)\end{array}$ & Grid & $\begin{array}{l}\text { Table } 3 \\
\text { Dataset } \\
\end{array}$ \\
\hline 1.1 & $\begin{array}{c}\text { Grid } \\
\text { Convergence }\end{array}$ & .85 & 5 & 100 & .5 & - & 0 & $\begin{array}{l}\mathrm{C} / \mathrm{M} / \\
\mathrm{F} / \mathrm{E}-\mathrm{F}\end{array}$ & $1 / 2 / 3 / 4 / 5$ \\
\hline 1.2 & Downwash & .85 & 5 & 100 & - & $\begin{array}{c}0 / 1 / 1.5 / 2 / \\
2.5 / 3 / 4\end{array}$ & $\begin{array}{c}\text { off } /-2 / \\
0 /+2\end{array}$ & $\mathrm{M}$ & $1 / 3$ \\
\hline 2 & Mach Sweep & $\begin{array}{c}.7 / .75 / .8 / .83 / \\
.85 / .86 / .87\end{array}$ & 5 & 100 & $\begin{array}{c}.4 / .45 / \\
.5\end{array}$ & - & 0 & M & 3 \\
\hline 3 & $\begin{array}{l}\text { Reynolds } \\
\text { Number }\end{array}$ & .85 & 20 & -250 & .5 & - & 0 & M & 3 \\
\hline
\end{tabular}

Table 4. Summary of DPW-IV Test Cases. 
Most of the information given in the above table is self-explanatory but a couple entries need clarification. First, Reynolds number (RN) is based on the wing reference chord length given in Table 1. Second, the extra-fine (E-F) grid for Test Case 1.1 was considered optional. The OVERFLOW datasets include E-F grid results but the CFL3D dataset does not. Following workshop guidelines, all cases were analyzed in free air with a fully turbulent boundary layer.

The overset grid CRM results are presented in two parts. The first part discusses results for all studies performed using the set of grids built before the workshop took place. These grids are available for download on the DPW-IV website (see Reference 6). The second part summarizes results from studies performed using grids built after the workshop. Both parts of this section include data obtained after the workshop.

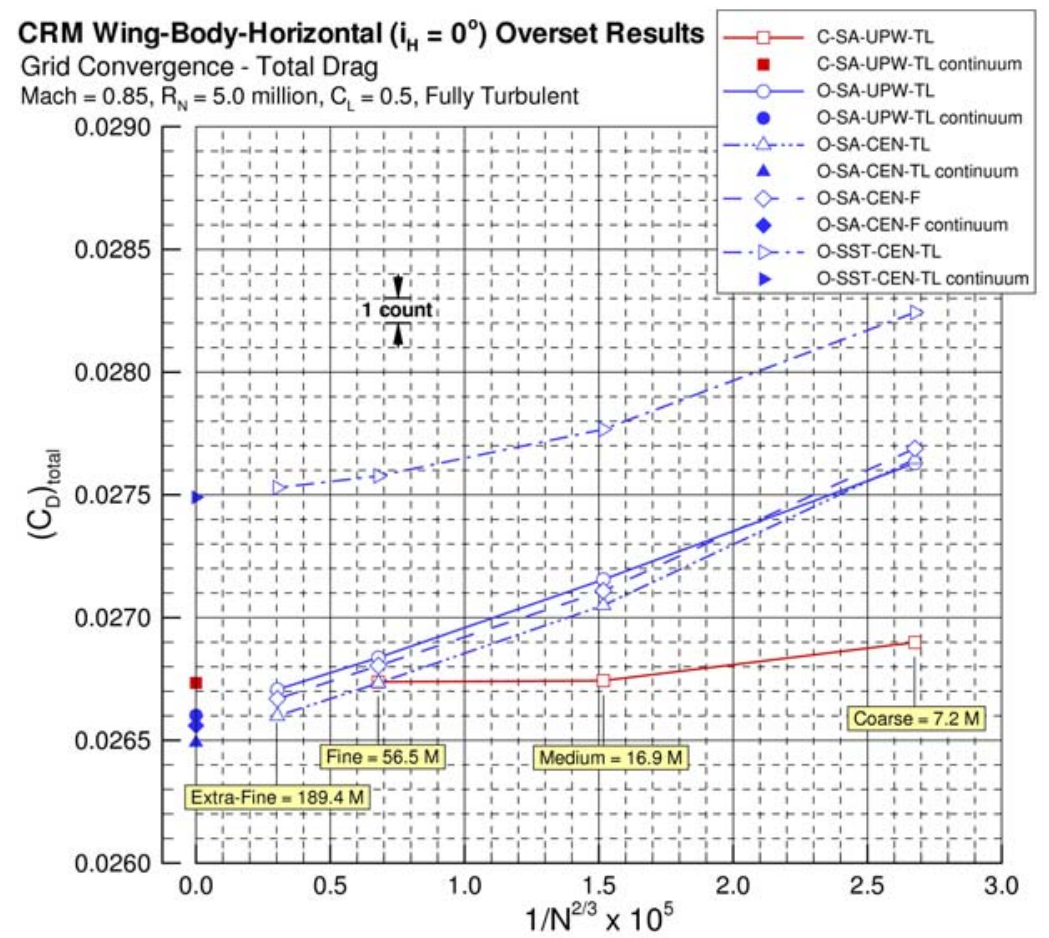

Figure 9. CRM Grid Convergence Study: Total Drag.

\section{A. Part I: Pre-Workshop Grid Studies}

The results discussed in this sub-section are from studies performed using the set of overset grids posted to the DPW-IV website. Some studies were completed prior to the workshop while others were performed as a follow-on exercise. This results summary will follow the order of test cases listed in Table 4 with emphasis on the grid convergence study and downwash study.

\section{Test Case 1.1 - Grid Convergence Study}

The grid convergence study focuses on grid-converged values of drag, pitching moment, and angle-of-attack for the wing-body-tail configuration at the design $C_{L}$ of 0.5 . The overset grid family used for this study is parametrically

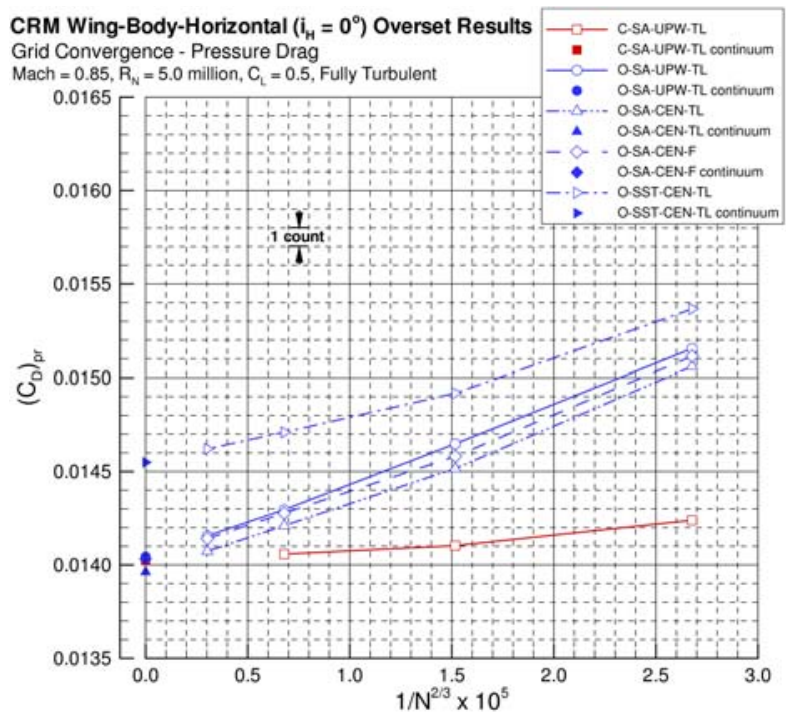

Figure 10. CRM Grid Convergence Study: Pressure Drag.

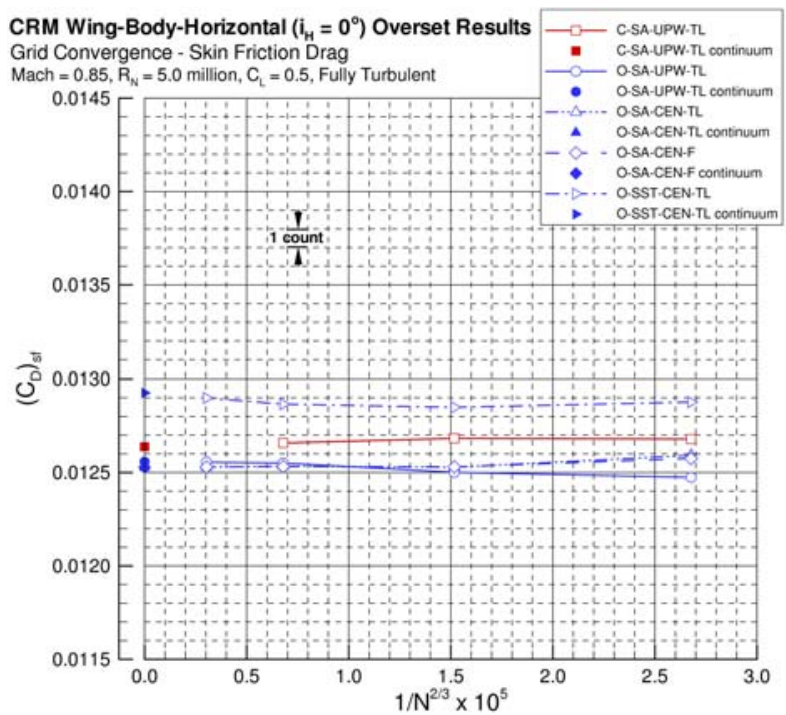

Figure 11. CRM Grid Convergence Study: Skin Friction Drag. 
equivalent (see Section III.B) and all grids were converged to the same level. Meeting these requirements allows for a meaningful application of Richardson extrapolation which, by assuming the method's accuracy, can be used to measure asymptotic grid convergence based on how linear the data plots. When the variable under study is plotted

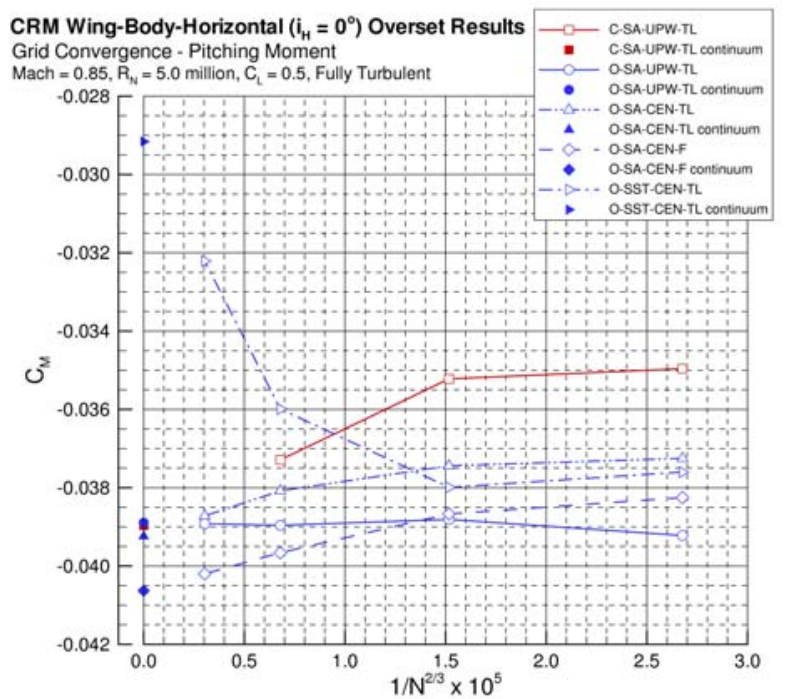

Figure 12. CRM Grid Convergence Study: Pitching Moment

are 5 counts and minor ticks are 1 count. If the coarse grid data is excluded, all datasets exhibit trends close to linear. The CFL3D-computed drag from the medium/fine grids are essentially the same whereas the OVERFLOWcomputed drag from the medium/fine/extra-fine grids decrease as the grid is refined. This makes for an interesting comparison of data between the engineeringgrade medium mesh and the extrapolated values (solid symbols). Comparing the data connected by solid lines where the two codes were run in a similar way (SA-UPW-TL), the 4.1 count drag difference between medium grid data decreases to 1.3 counts at the continuum. This is considered a very small code-to-code difference on the
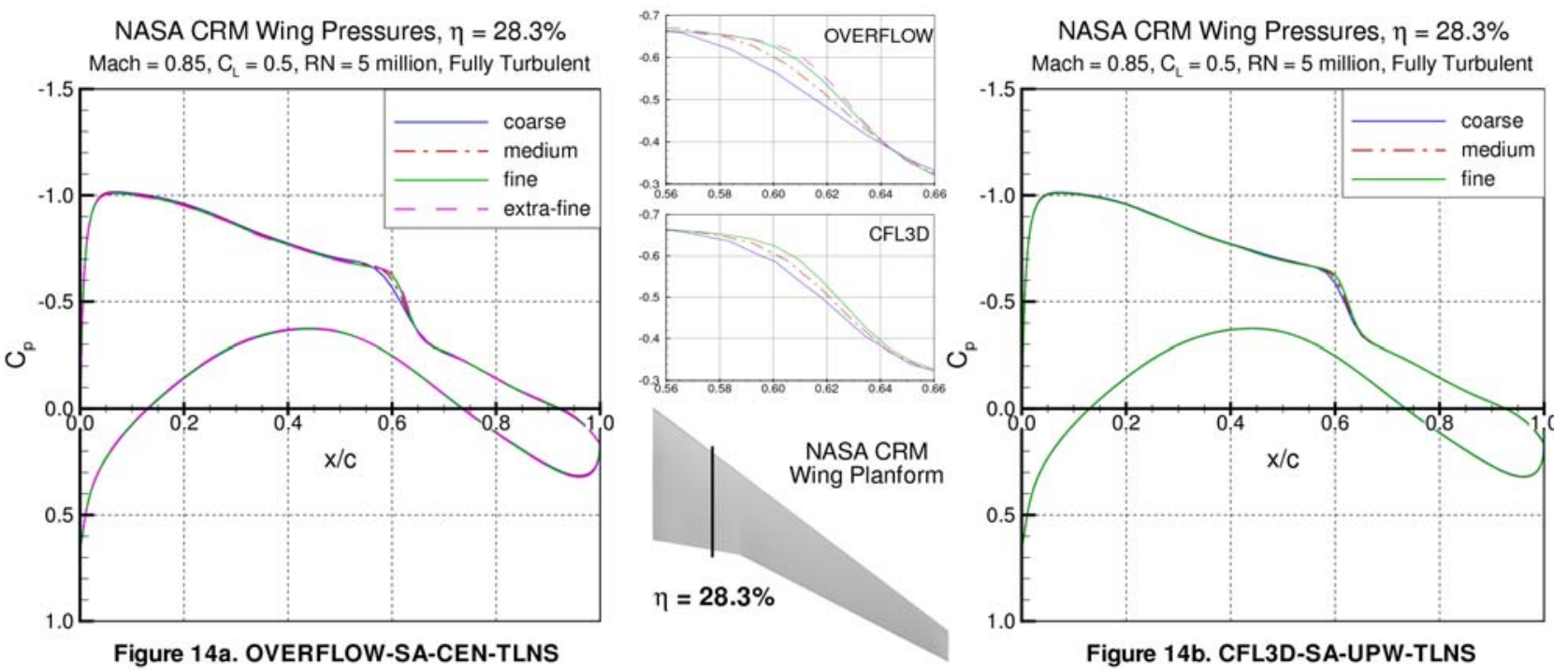

Figure 14. CRM Grid Convergence Study: Wing Pressure Comparison at $\eta=28.3 \%$.

American Institute of Aeronautics and Astronautics 
absolute level of drag and illustrates how well these two workhorse RANS codes compare when discretization error is eliminated. The SA, medium grid drag varies between 267.4 and 271.5 counts. The spread in SA data is reduced at the continuum where the minimum level is 265.6 counts and the maximum is 267.3 counts. The CEN-TL OVERFLOW results for the two different turbulence models show an increase in drag of about 7 counts for the

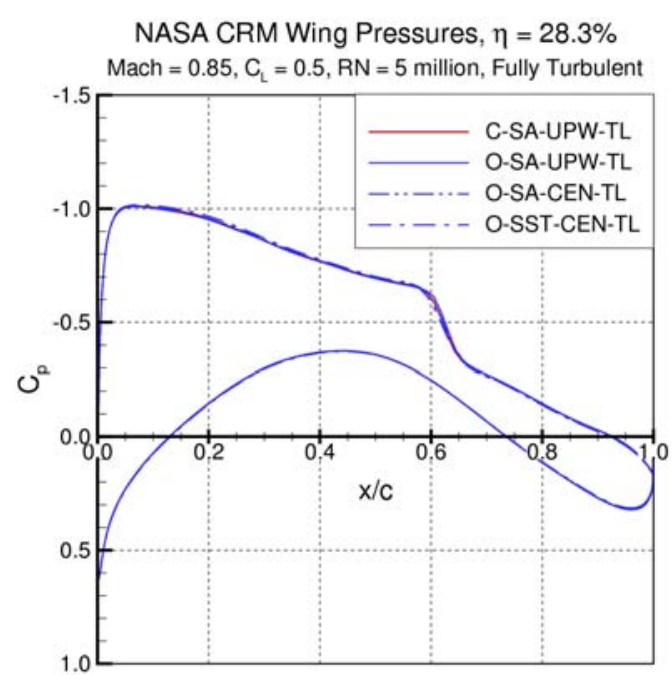

Figure 15a. Medium Grid Comparison at $\eta=28.3 \%$
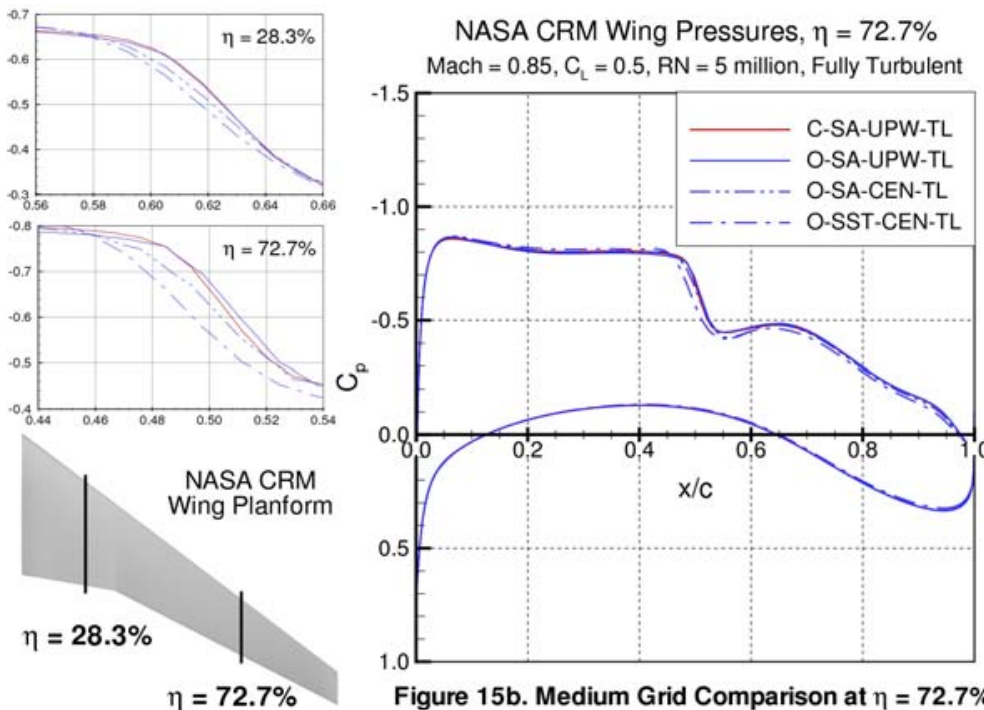

Figure 15b. Medium Grid Comparison at $\eta=72.7 \%$

Figure 15. CRM Medium Grid Wing Pressure Comparison at $\eta=28.3 \%$ and $72.7 \%$.

medium mesh with SST producing more drag than SA. Finally, the SA-CEN OVERFLOW data shown in Figure 9 indicate running the code using full Navier-Stokes will increase the total drag level by about 1 count for all grid sizes analyzed.

The components of drag are plotted in Figures 10 (pressure) and 11 (skin friction). Note the vertical axis in these two plots uses the same scale increments as in Figure 9. As seen in previous workshops, the general trends in pressure drag are similar to total drag. The SA pressure drag results plotted in Figure 10 extrapolate to a very tight cluster at the continuum with roughly a 1 count spread. Skin friction drag does not vary with grid size by more than

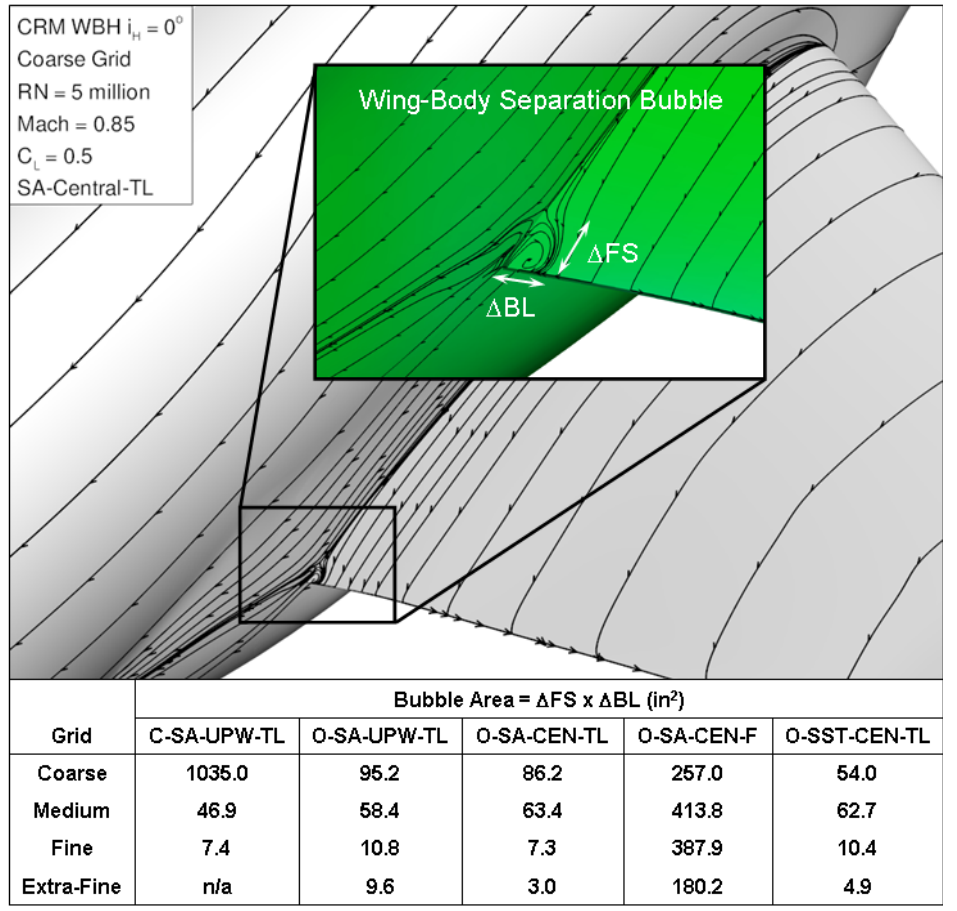

Figure 16. CRM Wing-Body Separation Bubble. 1 count for all datasets plotted in Figure 11. The OVERFLOW SA skin friction drag is nearly identical for the medium/fine/extrafine grids. CFL3D SA skin friction drag is slightly higher ( $\sim 1$ count) than OVERFLOW SA. The SST data represents the highest level of skin friction with a 3 to 4 count increase relative to the corresponding SA results.

CRM pitching moment results are summarized in Figure 12. Aircraft pitch was computed about the quarter-chord of the MAC. Excluding SST results and the coarse grid data, all datasets form close-to-linear trends indicating more nose-down pitching moment as the grid is refined. The medium/fine/extra-fine SST data is very nonlinear with an estimated pitching moment at the continuum of -0.03 . Relative to the SA data, the SST continuum moment is nose-up by about 0.01 . This significant difference is partially due to how the turbulence models predict flow separation at the tail-body juncture. More information on aft body flow is given later in this section where surface streamline images are compared. 


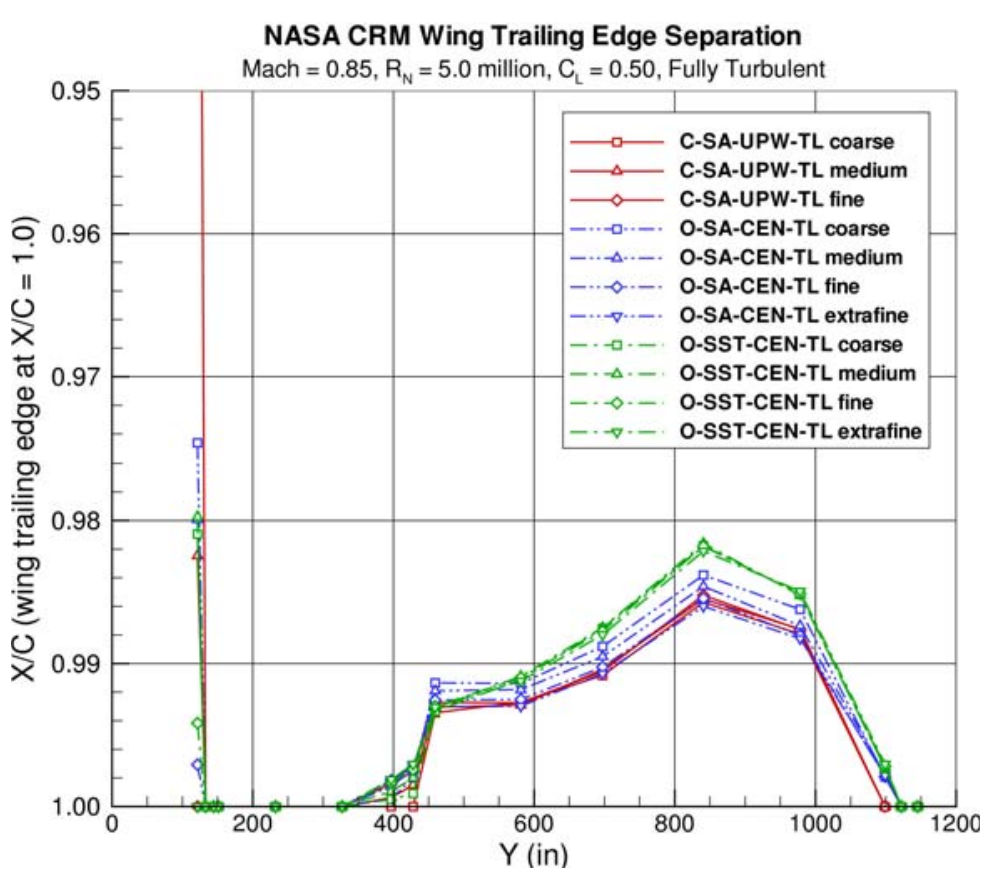

Figure 17. CRM Wing Trailing Edge Separation.
The angle-of-attack required for $\mathrm{C}_{\mathrm{L}}=$ 0.5 is compared in Figure 13. Both sets of SA-UPW-TL data agree very well with CFL3D requiring about 0.01 degrees more alpha than OVERFLOW. The SA-CEN OVERFLOW data extrapolated to the continuum represent the lowest alpha at roughly 2.287 degrees. The SST-CEN-TL OVERFLOW data gave the highest continuum alpha at 2.395 degrees. All datasets show reduced angle-of-attack for constant lift as the grid is refined.

Wing pressure comparisons are shown in Figure 14 with OVERFLOW coarse/medium/fine/extra-fine grid data plotted on the left (Figure 14a) and coarse/medium/fine grid CFL3D data on the right (Figure 14b). At this semi-span location of $28.3 \%$, the $\mathrm{C}_{\mathrm{p}}$ distribution varies with grid density at the shock only. Both codes show the same trend where more grid points appear to improve shock definition. The largest difference in $\mathrm{C}_{\mathrm{p}}$ is between the coarse and medium grid solutions which may help explain why the coarse grid drag data does not fall on a linear trend formed by the denser grid results. The comparisons made in Figure 14 are very similar to past workshops where the same $C_{p}$ variation with grid density was observed across the span of the wing. Therefore, it is reasonable to assume the effect of grid on wing pressures is adequately represented by the comparison at $28.3 \%$ semi-span. Figure 15 compares wing pressures at two semi-span stations for all of the TLNS datasets. FNS wing pressures are left out of this comparison because they are nearly identical to the TLNS results. Pressures at $28.3 \%$ semispan are plotted in Figure 15a (left side), and pressures at $72.7 \%$ semi-span are shown in Figure 15b (right side). Both Figures 14 and 15 include plots located in the center of the figure comparing pressures over a smaller scale. These center-plot comparisons show more details at the shock location. It is difficult to see any $C_{p}$ difference in Figure 15a between the various results, but when the scale is adjusted to include just the data at the shock, the differences are clear. When CFL3D and OVERFLOW are run using the same setup (SA-UPW-TL), the wing pressures at $28.3 \%$ semi-span are nearly identical. Comparing just the OVERFLOW results at this wing station shows turbulence modeling has more of an effect on shock prediction than the differencing schemes being employed. This observation is easier to make when looking at the outboard wing comparison in Figure $15 \mathrm{~b}$. The SST $\mathrm{C}_{\mathrm{p}}$ distribution is different than the corresponding SA data with an increased level of suction pressure going

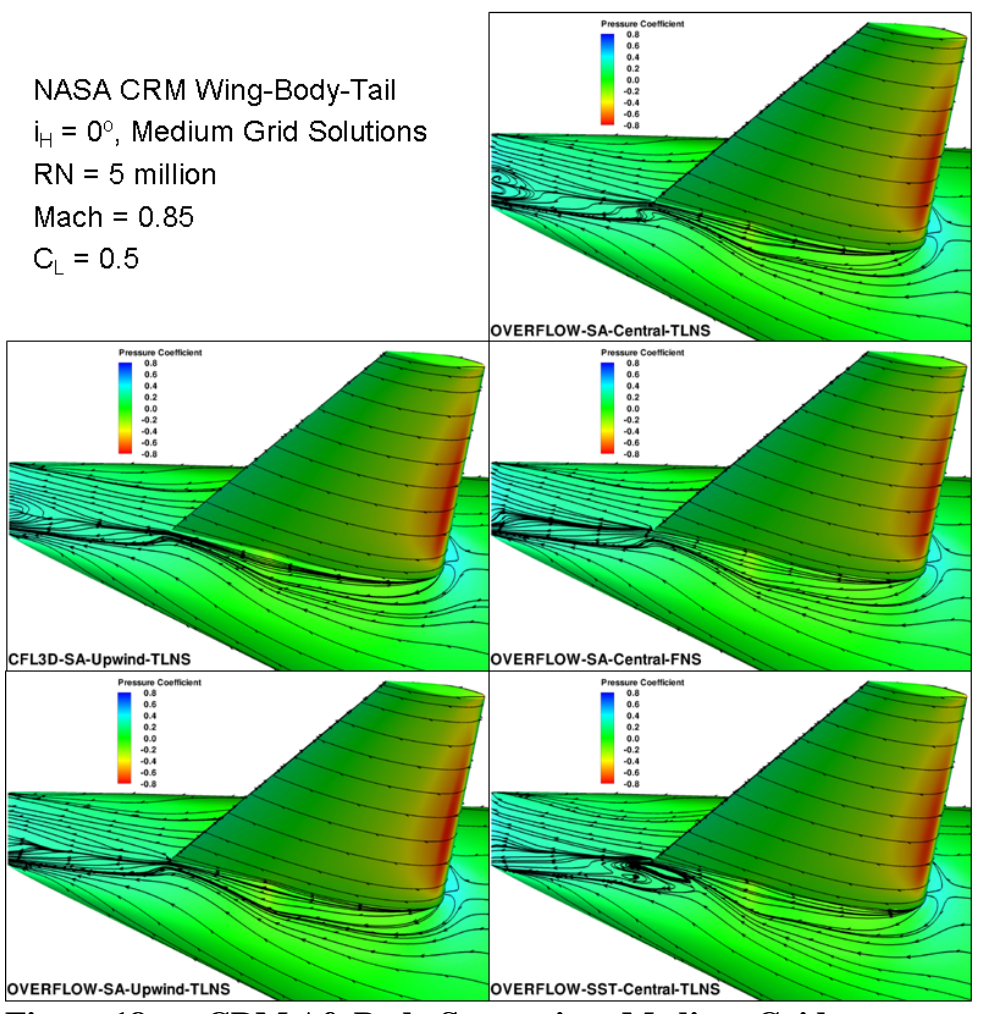

Figure 18. CRM Aft Body Separation: Medium Grid. 


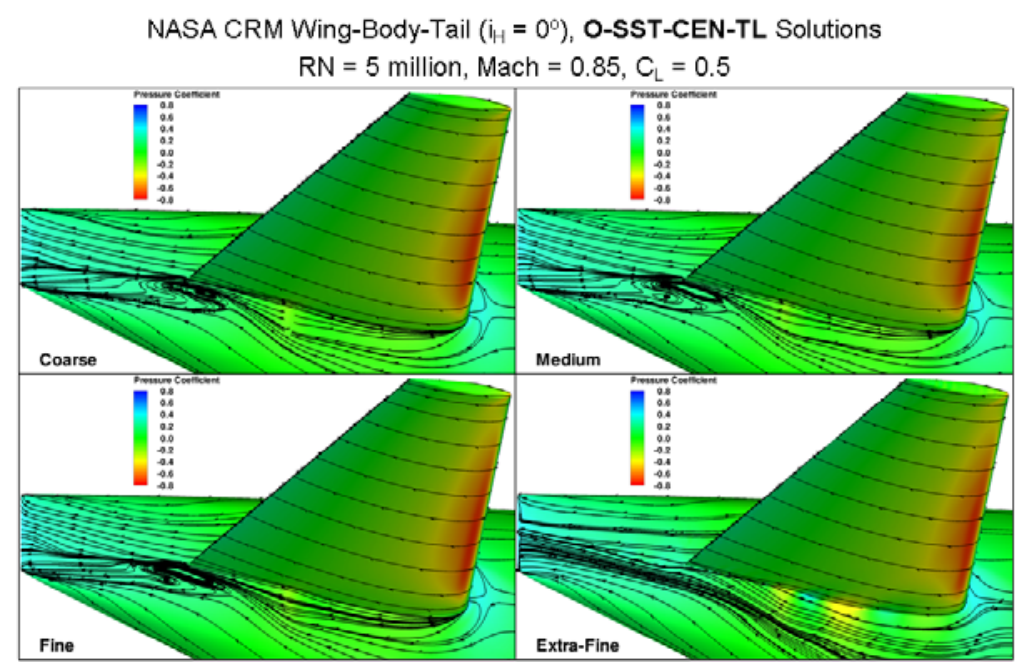

Figure 19. CRM Aft Body Separation: SST Solutions. into the shock and a reduced level aft of the shock. The SST turbulence model also predicts a shock location forward of the SA model by about $2 \%$ of the local chord on the outboard wing but a similar location inboard. This means the SSTcomputed shock has less sweep. A stronger shock with reduced sweep will result in higher shock drag and a weaker boundary layer downstream of the shock which helps explain why the SST drag levels are higher than SA (see Figure 9). As with the inboard wing comparison, the codes compute nearly identical pressures at $72.7 \%$ semi-span when run the same way.

Flow separation has always been an interesting topic of discussion for the DPW series. For DPW-IV, three areas of

separated flow were studied: 1) wing-body separation bubble at the wing's upper surface trailing edge, 2) wing and tail trailing edge separation, and 3) aft body separation in the tail-body juncture region and on the body's tailcone. Like the pressure comparisons discussed in the previous paragraph, surface flow features are studied for the configuration and condition specified in Test Case 1.1 (see Table 4). The CRM's wing-body juncture flow on the upper surface of the wing is characterized by the surface streamline images shown in Figure 16. The larger, underlying grey image in this figure shows streamlines on a portion of the body and inboard wing for the coarse grid O-SA-CEN-TL solution. The streamlines in the grey image highlight just how small the wing-body separation bubble is relative to the wing root chord ( $2.5 \%$ of the root chord). This is considered an acceptable amount of flow separation for this region at the cruise design condition at wind tunnel Reynolds numbers. The planform size of the wing-body separation bubble is estimated by multiplying its maximum length $(\Delta \mathrm{FS})$ by its maximum width $(\Delta \mathrm{BL})$. The inset image in Figure 16 defines these two lengths for the coarse grid solution. The resulting separation bubble area is tabulated at the bottom of Figure 16. All of the SA-TL bubble areas are reduced as grid density is increased with fine and extra-fine grid bubble sizes approaching zero. With the exception of the CFL3D coarse grid solution, there is good agreement between flow solvers. The FNS bubble sizes are significantly larger than TLNS which is an observation made in past workshops. This size difference helps explain why the FNS pressure drag is higher than TLNS. Finally, there is little variation in bubble area due to different turbulence modeling. Both the SA and SST streamlines look similar in the wingbody region.

Horizontal tail trailing edge separation was not found in any of the overset results, so only wing trailing edge separation is summarized in Figure 17 for the C-SA-UPW-TL (red), O-SACEN-TL (blue), and O-SST-CEN-TL (green) solutions. In this comparison, separation is defined as the point where the velocity vector is parallel to the wing trailing edge in the planform (z component ignored). The first grid plane off the surface was used to interrogate velocity vectors across the span of the

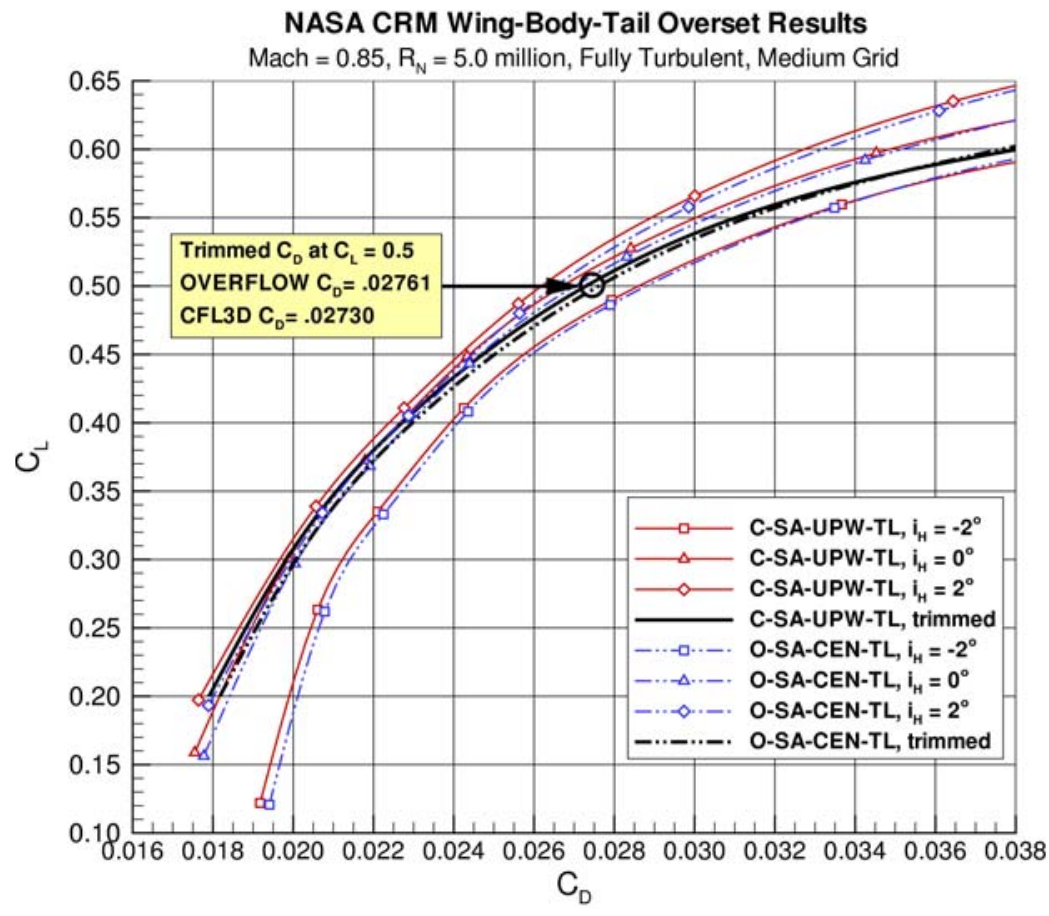

Figure 20. CRM Drag Polar: CFL3D vs. OVERFLOW. 


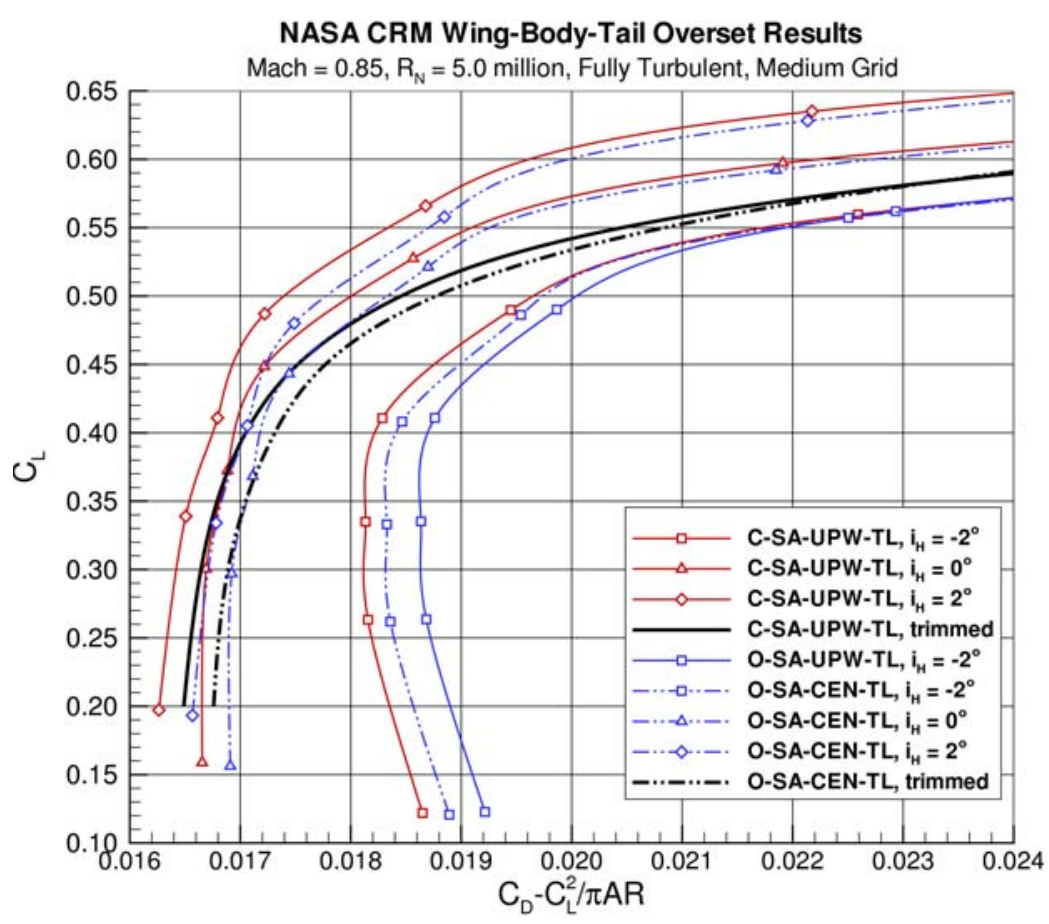

Figure 21. CRM Idealized Drag Polar: CFL3D vs. OVERFLOW. wing on the upper surface only. Some of the results plotted in Figure 17 show separation on the inboard wing represented by a spike at the side-ofbody. This is the wing-body separation bubble defined in Figure 16. Outboard of the separation bubble and inboard of the yehudi break $(\mathrm{Y}=428$ in), the overset solutions show little-to-no trailing edge separation. For the most part, wing trailing edge separation exists between the yehudi break and the wingtip, with the maximum separation occurring near $\mathrm{Y}=850 \mathrm{in}$ where it is $1.5 \%$ to $2 \%$ local chord. Comparing trends in separation chord extent, the CFL3D data show a very small difference with grid density with the largest variation occurring at the yehudi break. The OVERFLOW-SA results appear to be the most sensitive to grid refinement with a small reduction in separation $(\sim 0.3 \%)$ seen between the coarse and extra-fine grids. The SST turbulence model

predicts roughly $0.5 \%$ more trailing edge separation than SA.

Surface streamlines on the aft portion of the body at the tail-body intersection and tailcone are shown in Figures 18 and 19. With the exception of the SST dataset, the aft body surface flow features do not change with grid density so only the medium grid streamlines are compared in Figure 18. Two of the five medium grid solutions show aft body separation. The O-SA-CEN-TL streamline image shows a pocket of separated flow on the tailcone. This area of separation was not found in the corresponding FNS solution. The O-SST-CEN-TL streamline image also shows a pocket of separated flow but it is located near the horizontal tail's trailing edge. The SST aft body separation is examined further in Figure 19 where a variation with grid density can be seen. The lower portion of the separation pocket is not well defined in the coarse grid solution, and the extra-fine grid streamlines show no separation at the tail's trailing edge. Attached flow near the tail's root section should improve its effectiveness and this is exactly what the pitching moment data show in Figure 12. The SST extra-fine grid solution produces the most nose-up pitching moment indicating the tail is lifting downward more than in the other grid family solutions. The SST extra-fine grid surface pressure contours in Figure 19 show more variation on the lower surface of the tail at the side-of-body. This pressure variation is likely driven by improved definition of the geometry where the tail's scrubbing seal (flattened portion of the body) transitions to the tailcone. It is unclear if this local pressure variation and the absence of

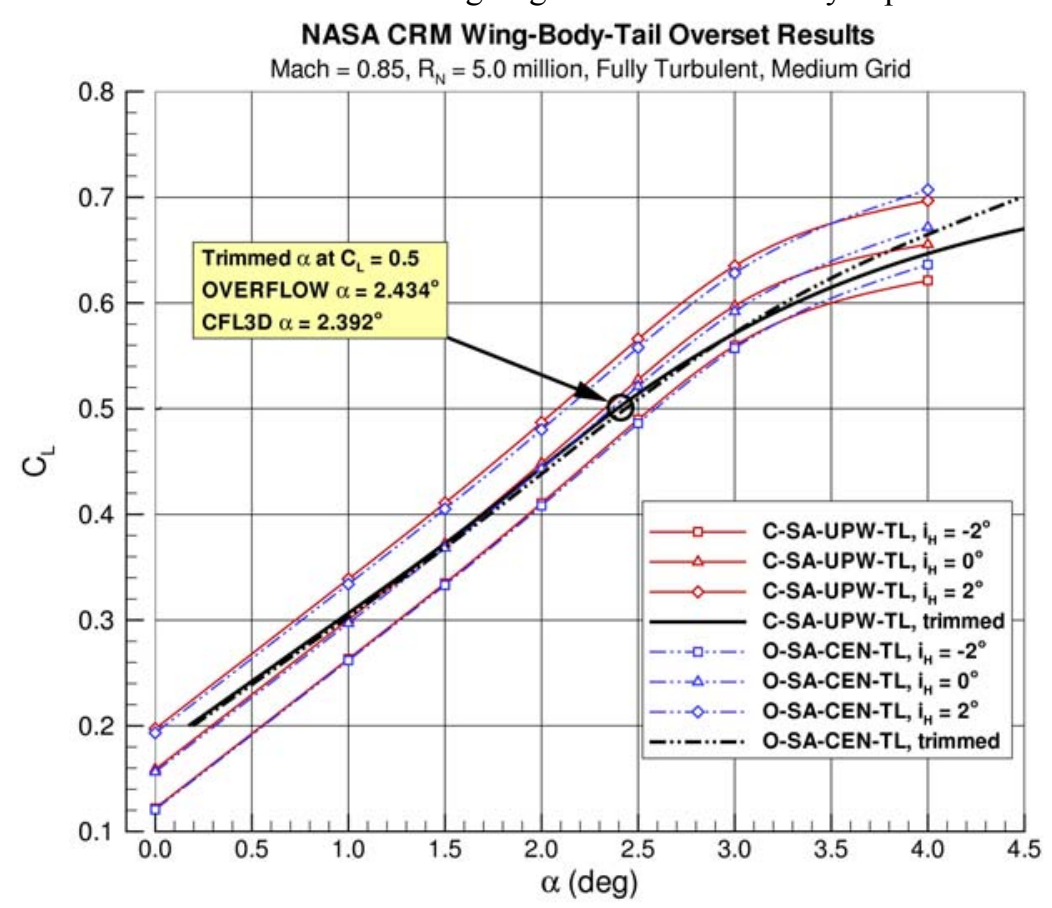

Figure 22. CRM Lift Curve: CFL3D vs. OVERFLOW. 
flow separation are related.

2. Test Case 1.2 - Downwash Study

The primary objective of the downwash study is to see how well CFD can predict a trimmed drag polar. To meet this objective, multiple tail settings were run through an alpha sweep at the design Mach of 0.85 and Reynolds number of 5 million (see Table 4). All runs were made on the medium mesh. The wing-body configuration was also analyzed to get a total drag penalty associated with trimming the CRM model. Only two of the five datasets summarized in Table 3 were available for the complete downwash study at the time this paper was written. These two datasets are from CFL3D (C-SAUPW-TL) and OVERFLOW (O-SA-CENTL), so code-to-code comparisons can be made. OVERFLOW was also run through an alpha sweep using upwind differencing (O-SA-UPW-TL) but only for the $-2^{\circ}$ tail setting. Limited code-to-code comparisons will be made between the two SA-UPWTL datasets.

CRM drag polars computed using the medium grid are compared in Figure 20. The red and blue curves with open symbols are data for the three tail settings analyzed. The black curves with no symbols represent the trimmed drag polars computed using an interpolation method provided by the DPW-IV organizing committee. By comparing curves with the same symbol, it is easy to see the two codes are in good agreement for all cases analyzed with CFL3D always predicting $\sim 3$ counts less drag than OVERFLOW. The difference is relatively constant, so the polar shapes are nearly identical. At the design $\mathrm{C}_{\mathrm{L}}$ of 0.5 , the CFL3D trimmed polar shows 3.1 counts less drag compared to OVERFLOW. Based on the drag comparisons made for the medium grid in Test Case 1.1 (Section V.A.1), the 3-to-4 count drag difference for a given configuration seen in Figure 20 will increase if both codes were run using upwind differencing (this will be discussed in more detail later). Even though the drag polars in Figure 20 were limited to a $\mathrm{C}_{\mathrm{L}}$ of 0.65 for comparison purposes, it is difficult to see differences at low $\mathrm{C}_{\mathrm{L}}$ 's for the $2^{\circ}$ and $0^{\circ}$ tail settings. An improved polar comparison can be made by removing idealized induced drag, $\mathrm{C}_{\mathrm{L}}{ }^{2} /(\pi \mathrm{e} A \mathrm{AR})$, with the Oswald efficiency number (e) set to 1 . The "idealized" profile drag is compared in Figure 21 over the same $C_{L}$ range used in Figure 20. The idealized profile drag comparison shows CFL3D and

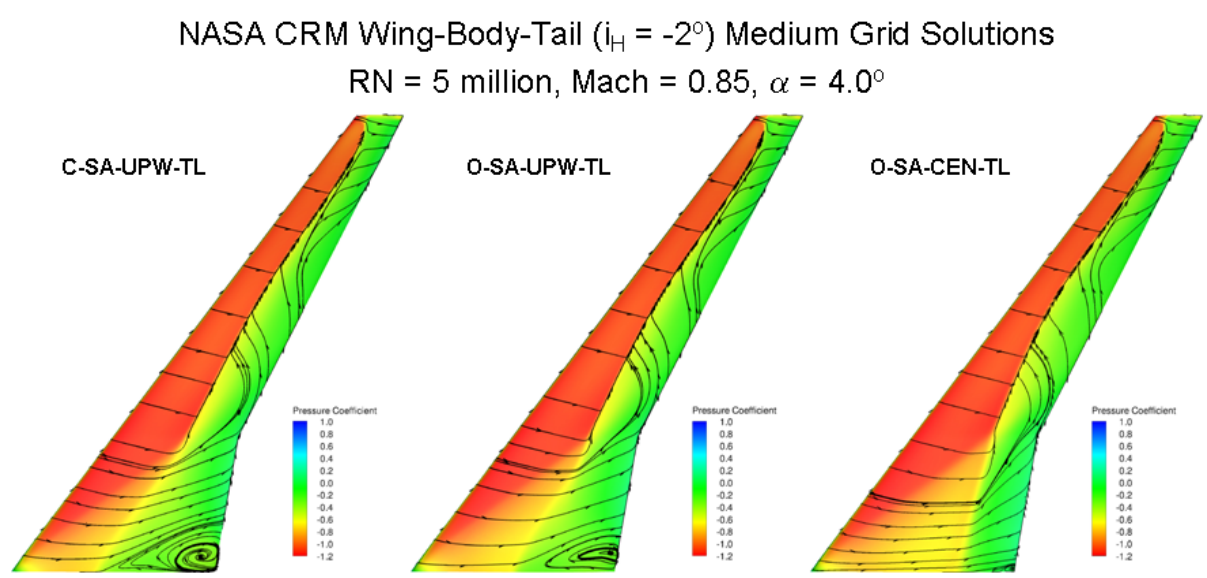

Figure 24. CRM Wing Streamlines: Effect of Differencing Scheme.

13

American Institute of Aeronautics and Astronautics
OVERFLOW are in good agreement for all cases analyzed with the largest difference in drag ( 4 counts) computed at low $\mathrm{C}_{\mathrm{L}}$.

The effect of differencing scheme on the idealized drag polar is shown in Figure 21 by comparing the SA-UPWTL results for the $-2^{\circ}$ tail. At low $\mathrm{C}_{\mathrm{L}}$ 's, the $\sim 3$ count drag difference between C-SA-UPW-TL and OSA-CEN-TL doubles to roughly 6 counts when 


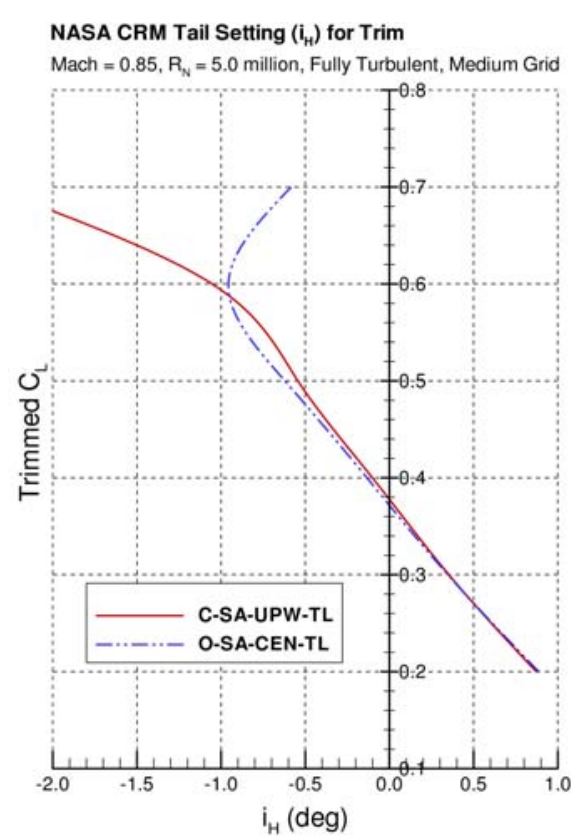

Figure 25. CRM Trimmed $i_{\mathrm{H}}$. the two codes use the same differencing scheme. This is a consistent trend with the medium grid $\mathrm{i}_{\mathrm{H}}=0^{\circ}$ results plotted in Figure 9; however, the magnitude of the drag difference between O-SA-CEN$\mathrm{TL}$ and O-SA-UPW-TL is larger for the $-2^{\circ}$ tail. Further analysis is required to fully understand these results.

Lift curve comparisons for the three tail settings and trimmed results are given in Figure 22. The codes predict nearly identical lift curves from $0^{\circ}$ to $3^{\circ}$ angle-of-attack. The lift curves break somewhere between $3^{\circ}$ and $4^{\circ}$. At $4^{\circ}$, CFL3D computes roughly 0.02 less $C_{L}$ than OVERFLOW. The reason for this will be discussed in more detail in the next paragraph. The alpha difference at the design $C_{L}$ of 0.5 is $0.04^{\circ}$.

Results for pitching moment are plotted in Figure 23. The moment reference center used to compute pitching moment is the quarter-chord of the wing's MAC (see Table 1). CFL3D and OVERFLOW are in general agreement on pitching moment for alphas of $0^{\circ}$ to $3^{\circ}$. The pitch break also compares well in that both codes predict a break near $3^{\circ}$ angle-of-attack. However, there is disagreement on the direction of the break with C-SA-UPW-TL breaking in the nose-down direction for tail settings of $0^{\circ}$ and $-2^{\circ}$ and O-SA-CEN-TL breaking nose-up for all three tails analyzed. Comparing results for $\mathrm{i}_{\mathrm{H}}=-2^{\circ}$ reveals an interesting trend with differencing scheme. When both codes are run with Roe upwind differencing, they agree on the direction of the pitch break (nose-down). The wing upper surface streamline comparison made in Figure 24 helps explain the different pitch break directions. Both CFL3D and OVERFLOW predict a large wing-body separation bubble at $4^{\circ}$ angle-ofattack using upwind differencing. When OVERFLOW was run with central differencing, the size of the wing-body separation was dramatically reduced. The larger wing-body separation bubble reduces lift on the inboard wing more than the shock induced separation does on the mid-wing. This results in a nose-down pitching moment. When the wing-body separation bubble is negligible, the inboard wing lift over-powers the rest of the wing causing the noseup pitch break seen in Figure 23. The differences seen in the $4^{\circ}$ solutions are a reminder of just how uncertain CFD results can be at extreme conditions where a significant amount of flow separation exists. The second part of the Results section (V.B.1) includes more discussion on this subject.

The tail setting required to trim the CRM wing-body-tail configuration at Mach 0.85 is shown in Figure 25. This plot compares tail settings computed using the medium grid results over a range of $\mathrm{C}_{\mathrm{L}}$ for both CFL3D and OVERFLOW. The influence of pitch break can be seen at the higher $\mathrm{C}_{\mathrm{L}}$ 's where CFL3D shows more negative $i_{H}$ is required to trim-out the increased nose-down pitching moment. The OVERFLOW results plotted in Figure 25 are from the dataset where central differencing was used, so the nose-up pitch break shown in Figure 23 requires a less negative tail setting for trim. At the design $C_{L}$ of 0.5 , CFL3D gives a trimmed $i_{H}$ of $-0.54^{\circ}$ while OVERFLOW predicts $-0.62^{\circ}$. So, despite the large differences in pitching moment at high $\mathrm{C}_{\mathrm{L}}$, the two codes are in good agreement at the design condition.

\section{Test Case 2 - Mach Sweep Study}

The second test case for DPW-IV was a Mach sweep study for the wing-body-tail configuration using the medium grid only. The $0^{\circ}$ tail setting was selected for this study.

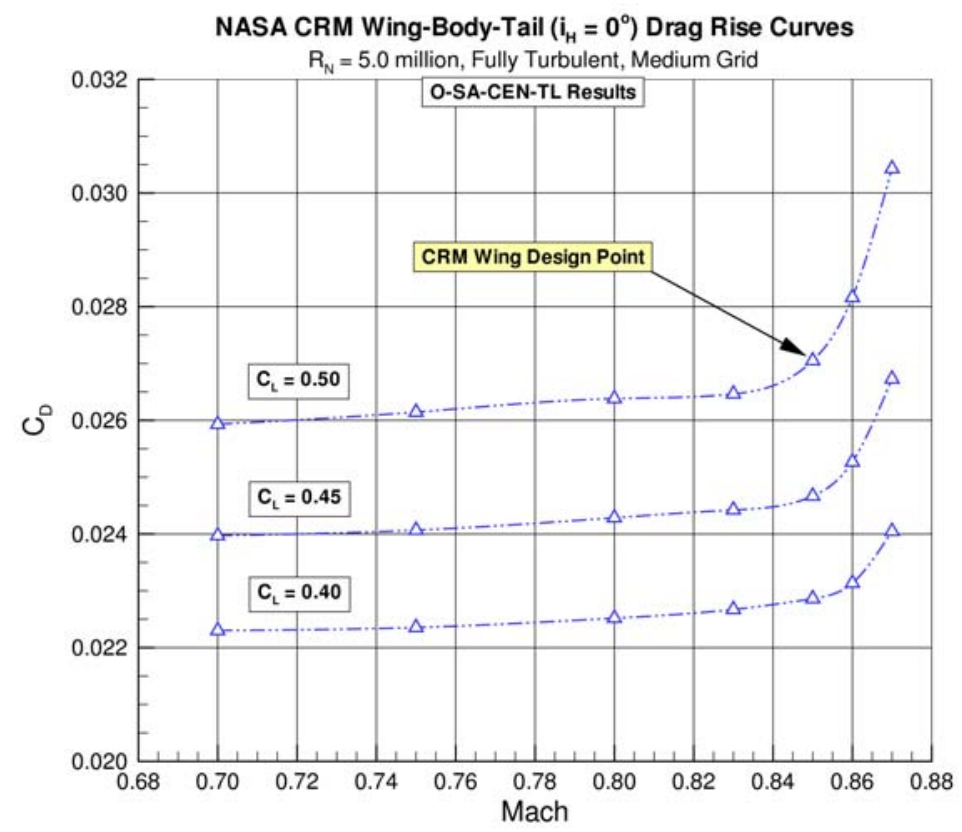

Figure 26. CRM Drag Rise Curves. 
CFL3D Mach sweep results were not done, so code-to-code comparisons will not be made. Figure 26 summarizes the OVERFLOW results in the form of three drag rise curves for $\mathrm{C}_{\mathrm{L}}$ 's of $0.40,0.45$, and 0.50 . The curves look reasonable in that drag levels rise with increasing $C_{L}$ for a given Mach, and the curve's bend at lower Mach numbers as $\mathrm{C}_{\mathrm{L}}$ is increased. The bend in the curve is referred to as the "drag rise." The CRM wing design point is shown to be part-way up the drag rise for these tail-on results. The OVERFLOW data plotted in Figure 26 are compared to other CFD results in Reference 21.

\section{Test Case 3 - Reynolds Number Study}

As discussed in Reference 5, the CRM model will be used to collect data across a range of Reynolds numbers at the NASA National Transonic Facility (NTF) wind tunnel. Test Case 3 allows for the prediction of Reynolds number effects prior to the NTF test by comparing results from Test Case 1 to runs made at $\mathrm{RN}=20$ million. This comparison is limited to the wing-body-tail medium grid analyzed at the design condition. Since this is a single-point study, the results are best summarized in tabular form. As with the Mach Sweep Study, CFL3D results are not available, so a code-to-code comparison is not possible. The OVERFLOW results given in Table 5 are part of the O-SA-CEN-TL dataset. The angle-of-attack required to maintain the design $\mathrm{C}_{\mathrm{L}}$ of 0.5 is reduced by $0.18^{\circ}$ going from 5 million to 20 million RN. Drag is also reduced by 30.2 counts. Increased Reynolds number drives the pitching moment more nose-down indicating a slight outboard shift in wing loading and attached flow near the tail cone. The OVERFLOW Reynolds number increments given in Table 5 are

\begin{tabular}{|c|ccc|}
\hline \multicolumn{4}{|c|}{ Wing-Body-Tail $\left(\mathrm{i}_{\mathrm{H}}=0^{\circ}\right)$} \\
Medium Grid, Mach $=0.85, \mathrm{C}_{\mathrm{L}}=0.50$ \\
\hline $\mathrm{RN}$ & $\alpha(\mathrm{deg})$ & $\mathrm{C}_{\mathrm{D}}$ & $\mathrm{C}_{\mathrm{M}}$ \\
\hline \hline 5 mil & 2.364 & .02706 & -.0375 \\
$20 \mathrm{mil}$ & 2.183 & .02404 & -.0414 \\
$\Delta$ & -.181 & -.00302 & -.0039 \\
\hline
\end{tabular}
compared to other CFD results in Reference 21.

\section{B. Part II: Post-Workshop Grid Studies}

This second part of the results summary focuses on two continuation studies where additional OVERFLOW data are used to further explore some of the DPW-IV test case findings. First, the effect of local grid refinement on wing separation at high angle-of-attack is investigated. This is followed by an expanded grid convergence study where extreme global grid refinement is done to test conclusions made regarding asymptotic grid convergence and the use of Richardson extrapolation on the overset results. Both of these studies are done using grids built or modified after

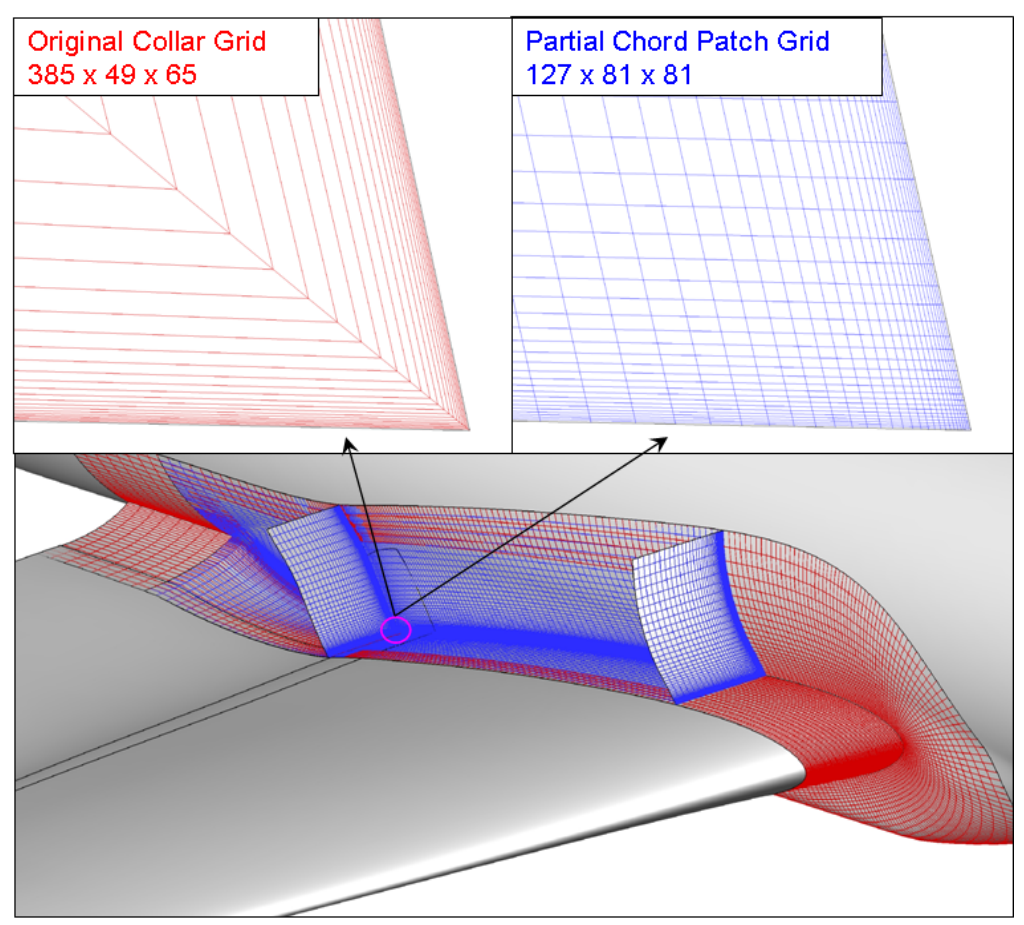

Figure 27. Wing-Body Collar Grid and Patch Grid. the workshop was held.

\section{Side-of-Body Patch Grid Study}

The purpose of this study is to see what effect local grid refinement has on the prediction of a wing-body separation bubble at high alpha. The extent of separation on this region of the configuration has a direct impact on high speed stall characteristics as illustrated in Figures 23 and 24. Accurate prediction of this type of flow separation begins with adequate grid resolution and quality. If grid density is not sufficient, then studies on turbulence modeling or TLNS vs. FNS may not produce meaningful conclusions. Concerns about high grid cell aspect ratios and high stretching ratios along the wing-body intersection was the primary motivation for replacing a portion of the original collar grid with a dense patch grid. Figure 27 compares a plane from each grid at the same location near the wing 
trailing edge. A grid plane from the collar grid is shown as the red image in the upper left portion of Figure 27 while the corresponding plane from the patch grid is colored blue on the right. When two components (like a wing and body) intersect, the grid topology typically used to define the intersection region is referred to as a collar. Depending on the surface point clustering at the corner, characteristics of the collar's volume grid can be an issue. Volume grid generators such as HYPGEN $^{13}$ march away for the concave corner in a way that sometimes results in poor local grid quality (i.e. excessive stretching). The high cell aspect ratio seen in the upper left insert image in Figure 27 is another collar grid characteristic. In an attempt to better understand

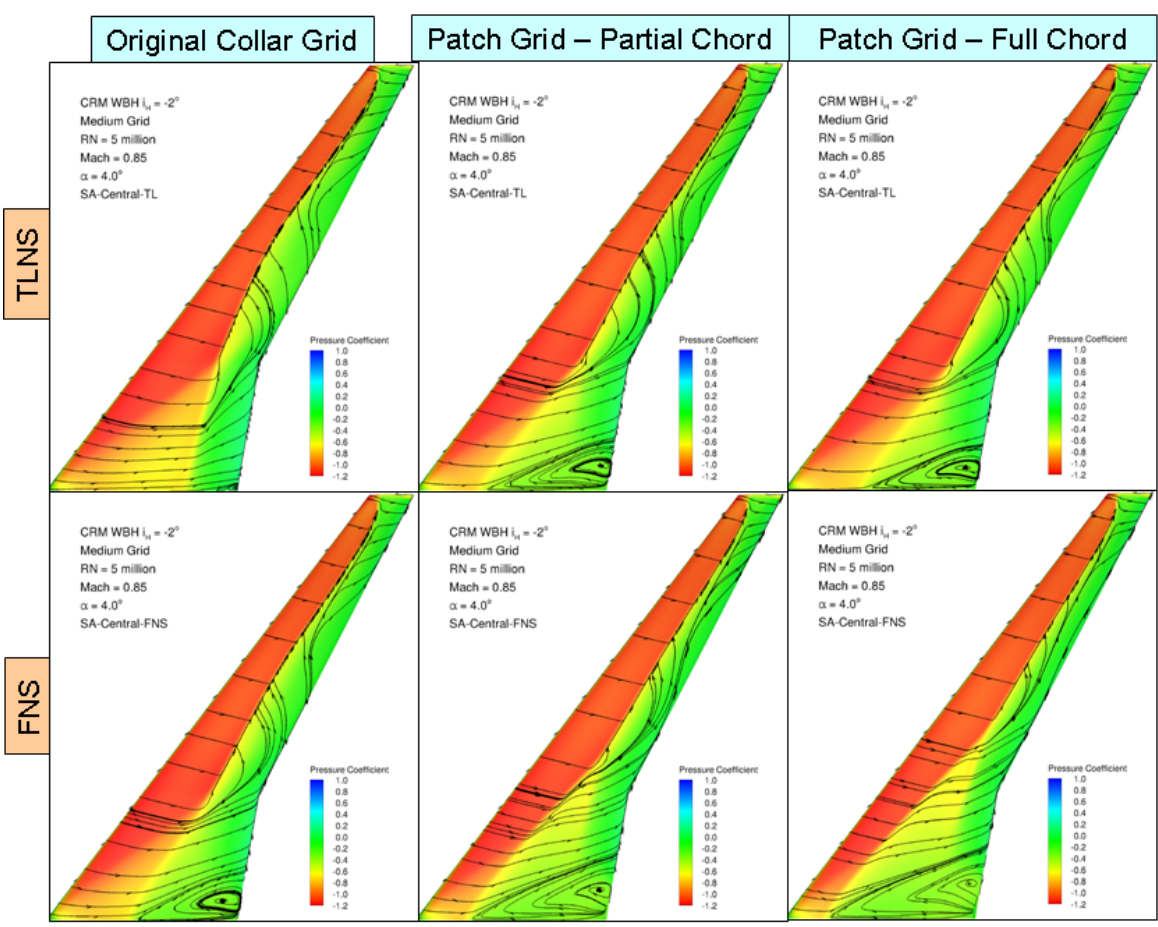

Figure 28. CRM Wing Streamlines at $\alpha=4^{\circ}$ : Patch Grid Effect. how these features of the wing-body collar grid impact flow separation, a purpose-built patch grid was analyzed. The patch grid has viscous clustering in two directions allowing the cell aspect ratio at the corner to be exactly one. Initially, a partial-chord patch grid was built and analyzed to limit grid refinement to the area of concern. When questions were raised about boundary layer interpolation at the faces of the patch grid, a full-chord version was constructed. For both patch grids, a hole is cut-out of the underlying collar grid to ensure the solver uses the desired topology.

Both the partial-chord and full-chord patch grids were analyzed using OVERFLOW with the Spalart-Allmaras (SA) turbulence model and central differencing (CEN). The code was run using both thin-layer and full NavierStokes at angles-of-attack of $3^{\circ}$ and $4^{\circ}$. Since the pitch break discrepancy was studied at a horizontal tail setting of $-2^{\circ}$, the patch grids were analyzed for this wing-body-tail configuration, and the results were interrogated for surface streamlines only. Based on upper surface wing streamlines, the extent of separation is relatively small at $3^{\circ}$ of alpha for the original collar grid solutions. Including the patch grid had no effect on the wing-body separation bubble. However, at $4^{\circ}$ where the separation was extremely large for the upwind solutions, the patch grid did have an impact. Figure 28 compares the alpha $=4^{\circ}$ results for the three grid topologies and two solution types. The top row of images in Figure 28 summarizes the TLNS runs while the bottom row represents the FNS runs. Qualitatively speaking, the partial-chord and full-chord patch grids have the same effect which is to drive the bubble size up dramatically. It is interesting to note that the FNS collar grid results look similar to the TLNS patch grid results which means the FNS solutions are less sensitive to local grid refinement. When these results are combined with the findings previously discussed regarding the effect of differencing scheme, a story emerges. The bubble size is clearly a function of many things including grid resolution, differencing scheme, and direction of viscous terms included. Adding more grid points, using upwind, or including more terms in the viscous flux calculation drives the solution towards an increase in bubble size, which is believed to be the wrong direction compared to experiment. However, the large bubble size appears to be the grid-converged result for this turbulence model (i.e., when discretization errors are adequately reduced). More work is needed to better understand these trends.

\section{Extended Grid Convergence Study}

The idea behind this study is simple - increase grid size beyond the extra-fine mesh to see if the near-linear drag trends summarized in Section V.A continue toward the continuum. Executing this study was anything but simple due to the extreme grid densities required. Knowing grid point count needed to be in the billions to make this study worthwhile, some initial planning was conducted where the Boeing and NASA Ames authors discussed issues such as solution file record length limitations and computing requirements. The plan that came out of these discussions 
called for the expanded set of grids to be built by Boeing using the same procedure followed for the workshop grids. The OVERFLOW analysis was to be completed at NASA Ames.

The first step in building the expanded set of grids was to address solution file record length limitations. A maximum record length of $2,147,483,648$ bytes was used to establish an upper bound on grid point count for a given zone in the largest grid. For the OVERFLOW solution file, 8 double-precision (64 bit or 8 byte) variables were assumed for each grid point. This means the solution file could contain 64 bytes of information at each point. Dividing the maximum record length of 2 gigabytes by 64 bytes/point gives an upper limit of 33.5 million points per zone. In order to meet this limitation, the 17 individual grids makingup the wing-body-tail configuration were broken into smaller pieces which drove the number of zones up to 81 for this expanded grid convergence study. This fundamental change in grid topology was expected to have an effect on the results, so new dataset names were assigned. The family of grids built for DPW-IV is referred to as "Grid Family 1" and the new, 81 zone family built for this follow-on study is referred to as "Grid Family 2". Figure 29 contains a side-by-side planform comparison showing surface grid edges for both grid topologies.

After the extra-fine grid from Grid Family 1 (GF1) was chopped-up into smaller pieces to form the extra-fine grid for Grid Family 2 (GF2), the process outlined in Section III.B was followed to increase grid size by a factor of

Table 6a. Summary of Grid Parameters for the 17 Zone Wing-Body-Tail.

\begin{tabular}{|c|c|c|c|c|c|c|}
\hline \multicolumn{7}{|c|}{ Grid Family 1-17 Zone Grid System } \\
\hline Grid & Points & $1 / \mathrm{N}^{2 / 3} \times 10^{5}$ & $1^{\text {st }}$ Cell Size & $\mathrm{y}^{+}$ & $\begin{array}{c}\text { Constant } \\
\text { Cells }\end{array}$ & $\begin{array}{c}\text { Growth } \\
\text { Rate }\end{array}$ \\
\hline Coarse & $7,221,233$ & 2.677 & .00104 in & .87 & 2 & 1.26 \\
Medium & $16,932,913$ & 1.517 & .00079 in & .66 & 3 & 1.19 \\
Fine & $56,531,489$ & 0.679 & .00052 in & .44 & 4 & 1.12 \\
Extra-Fine & $189,413,153$ & 0.303 & .00035 in & .29 & 6 & 1.08 \\
\hline
\end{tabular}

Table 6b. Summary of Grid Parameters for the 81 Zone Wing-Body-Tail.

\begin{tabular}{|c|c|c|c|c|c|c|}
\hline \multicolumn{7}{|c|}{ Grid Family 2-81 Zone Grid System } \\
\hline Grid & Points & $1 / \mathrm{N}^{2 / 3} \times 10^{5}$ & $1^{\text {st }}$ Cell Size & $\mathrm{y}^{+}$ & $\begin{array}{c}\text { Constant } \\
\text { Cells }\end{array}$ & $\begin{array}{c}\text { Growth } \\
\text { Rate }\end{array}$ \\
\hline Extra-Fine & $213,365,393$ & 0.280 & .00035 in & .29 & 6 & 1.08 \\
Super-Fine & $714,230,353$ & 0.125 & .00024 in & .20 & 10 & 1.05 \\
Ultra-Fine & $2,400,715,826$ & 0.056 & .00018 in & .15 & 19 & 1.04 \\
\hline
\end{tabular}

Table 6. CRM Wing-Body-Tail Grid Parameters for Extended Grid Study. roughly 3.4 (or $1.5^{3}$ ). The GF2 extra-fine grid was used to create a super-fine grid consisting of 714 million points (3.35 times greater than the extra-fine) and an ultra-fine grid consisting of 2.4 billion points (3.36 times greater than the super-fine). Table 6 compares various grid parameters between the two grid families with GF1 summarized in Table $6 \mathrm{a}$ and GF2 summarized in Table 6 b. Note the increase in point count for the extra-fine grid going from GF1 to GF2. This increase is a result of additional grid overlap.

The Boeing computing platform used for the GF1 OVERFLOW study (as described in Section IV.B) does not have the processing power required for GF2 grid densities, so resources at the NASA Advanced Supercomputing (NAS) facility were used for the GF2 OVERFLOW analysis.

The runs were made on the Pleiades supercomputer which has a total of 56,832 cores on 7,104 nodes. With the exception of the ultra-fine grid, the Pleiades runs were made on nodes consisting of 2 quad-core processors. Each of the 8 cores has $1 \mathrm{~GB}$ of memory. The number of cores for a given OVERFLOW run varied depending on grid size. 
Table 7 provides a breakdown on core count as well as general timing information for the two types of computing platforms utilized. Note, the ultra-fine grid was run on nodes with 8 cores as well as 12 cores. The 12 core run is summarized in the last row of Table 7.

\begin{tabular}{|c|c|c|c|c|c|c|c|}
\hline \multicolumn{8}{|c|}{ NASA CRM Wing-Body-Tail OVERFLOW Analysis on the Boeing PC Cluster } \\
\hline Grid & Cores & $\begin{array}{l}\text { CPU Time, T } \\
(\mathrm{sec})\end{array}$ & Steps, S & $\begin{array}{l}\mathrm{R}=\mathrm{T} / \mathrm{S} \\
\text { (sec/iter) }\end{array}$ & Grid Points, G & $\begin{array}{l}\mathrm{R} / \mathrm{G} \\
\text { (sec/iter/grid) }\end{array}$ & $\begin{array}{l}\text { Soln File } \\
\text { Size (GB) }\end{array}$ \\
\hline GF1 coarse & 16 & 18,000 & 4,000 & 4.5 & $7,221,233$ & $62.3 \times 10^{-8}$ & 0.40 \\
\hline GF1 medium & 16 & 50,400 & 4,000 & 12.6 & $16,932,913$ & $74.4 \times 10^{-8}$ & 0.95 \\
\hline GF1 fine & 32 & 106,560 & 4,000 & 26.6 & $56,531,489$ & $47.1 \times 10^{-8}$ & 3.17 \\
\hline GF1 extra-fine & 48 & 590,400 & 8,200 & 72.0 & $189,413,153$ & $38.0 \times 10^{-8}$ & 10.61 \\
\hline \multicolumn{8}{|c|}{ NASA CRM Wing-Body-Tail OVERFLOW Analysis on the NAS Pleiades Supercomputer } \\
\hline Grid & Cores & $\begin{array}{l}\text { CPU Time, T } \\
(\mathrm{sec})\end{array}$ & Steps, S & $\begin{array}{l}\mathrm{R}=\mathrm{T} / \mathrm{S} \\
\text { (sec/iter) }\end{array}$ & Grid Points, G & $\begin{array}{l}\mathrm{R} / \mathrm{G} \\
\text { (sec/iter/grid) }\end{array}$ & $\begin{array}{l}\text { Soln File } \\
\text { Size (GB) }\end{array}$ \\
\hline GF1 coarse & 32 & 11,049 & 8,210 & 1.34 & $7,221,233$ & $18.0 \times 10^{-8}$ & 0.40 \\
\hline GF1 medium & 128 & 13,102 & 16,000 & 0.82 & $16,932,913$ & $4.8 \times 10^{-8}$ & 0.95 \\
\hline GF1 fine & 128 & 29,448 & 10,000 & 2.95 & $56,531,489$ & $5.2 \times 10^{-8}$ & 3.17 \\
\hline GF1 extra-fine & 1024 & 65,953 & 40,000 & 1.65 & $189,413,153$ & $0.87 \times 10^{-8}$ & 10.61 \\
\hline GF2 extra-fine & 1024 & 18,952 & 10,000 & 1.89 & $213,365,393$ & $0.89 \times 10^{-8}$ & 11.95 \\
\hline GF2 super-fine & 2048 & 35,460 & 10,000 & 3.55 & $714,230,353$ & $0.49 \times 10^{-8}$ & 40.00 \\
\hline GF2 ultra-fine & 4096 & 62,677 & 8,000 & 7.83 & $2,400,715,826$ & $0.33 \times 10^{-8}$ & 134.44 \\
\hline GF2 ultra-fine & 4104 & 30,487 & 6,000 & 5.08 & $2,400,715,826$ & $0.21 \times 10^{-8}$ & 134.44 \\
\hline
\end{tabular}

Table 7. Timing and File Size Information for the CRM OVERFLOW Analysis.

The ultra-fine grid was run on a total of 4096 cores using the dual quad-core nodes with 8 GB of memory. This was set by memory requirements, so the GF2 runs do not represent optimum parallel performance. In general, the time per iteration listed in Table 7 show Pleiades to be about one order of magnitude faster than the PC cluster. These numbers are based on wall clock time where I/O is included. The column on the right of Table 7 shows how much the OVERFLOW solution file grows with grid size. The solution file for the 2.4 billion point grid is $134.4 \mathrm{~GB}$ which is almost 142 times larger than the medium grid solution file.

OVERFLOW version 2.1ae was run on Pleiades using the same setup as that outlined for Dataset \#3 in Table 3. The name assigned to this set of data is O-SA-CEN-TL. The one difference in the way the code was run has to do with the use of multi-grid for convergence acceleration. When the super-fine and ultra-fine grids were built, no attempt was made to maintain multi-grid levels greater than one. This was thought to be acceptable because OVERFLOW is designed to run on such grids, but convergence problems prevented the larger GF2 grids from being run with multi-grid turned on. For this reason, the number of steps listed in Table 7 is considerably larger for Pleiades.

GF1 was re-analyzed at NASA Ames to get a consistent set of data for the extended grid convergence study. Since the exact same grid was run using the same code setup (SA-CEN-TL), the Boeing and NASA Ames GF1 results were expected to be very close. Figure 30 compares total drag from these two sets of GF1 data. The coarse and medium grid results compare very well with total drag varying by roughly 0.1 counts. The difference grows slightly between the medium and fine grids and again between the fine and extra-fine grids where total drag is roughly 0.5 counts higher for the NASA Ames solution. There are at least three sources for the GF1 drag differences: 1) different code version, 2) different method for pressure integration used to get forces and moments, and 3) different levels of convergence. The first two items were thoroughly investigated and found to have a minimal drag impact of less than 0.1 counts. Since convergence becomes more challenging for higher grid densities, the 0.1 count drag difference for the coarse and medium grid results is most likely due to items 1 and 2 . The larger difference for the fine and extra-fine grids is believed to be a combination of all three items with convergence levels contributing the most. Both sets of GF1 total drag data appear to be in the range of asymptotic grid convergence if the coarse grid data is ignored. 
The green data points plotted in Figure 30 represent the NASA Ames GF2 results. For the scale used to compare these drag results, it looks like the GF2 data form a continuation of the GF1 fine and extra-fine trend toward the continuum. This was unexpected given the increased amount of grid overlap introduced in the 81 zone GF2 topology (see Figure 29). The expectation that such a large difference in overlap between GF1 and GF2 would cause

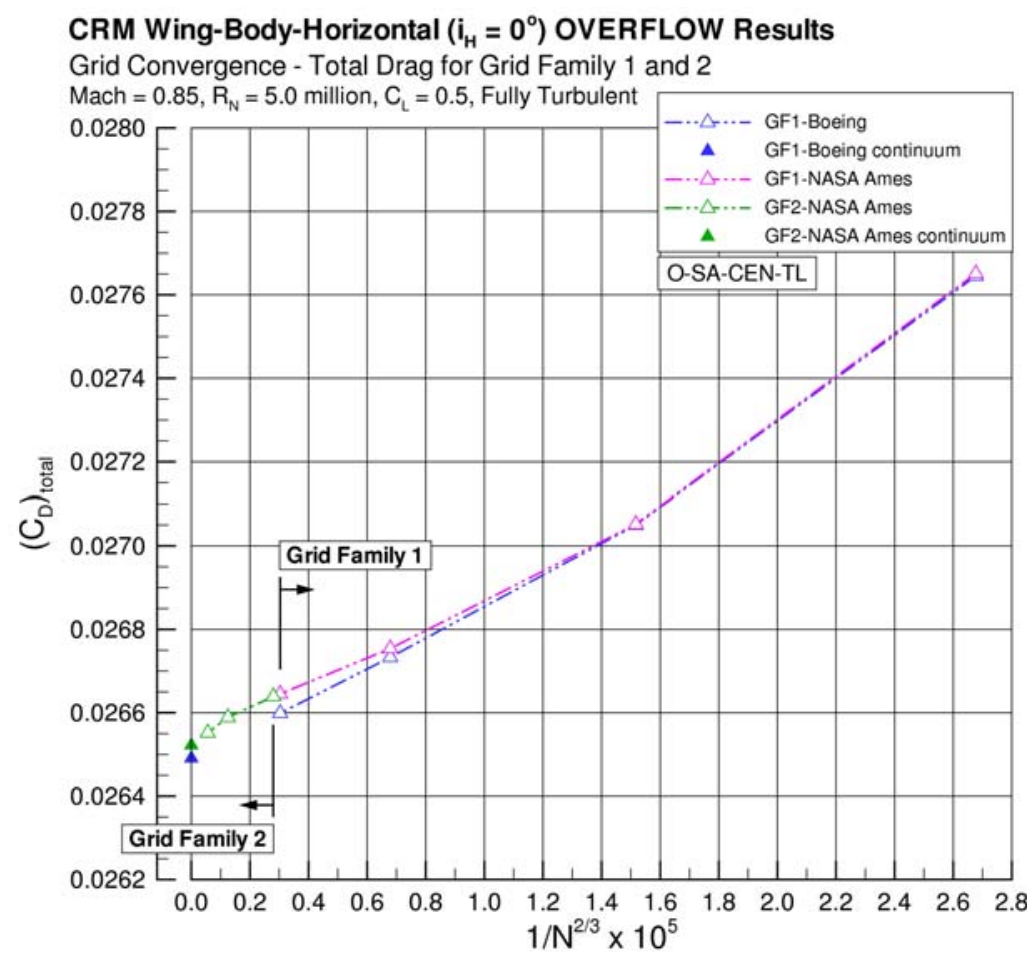

Figure 30. CRM Extended Grid Convergence Study: Total Drag. a significant shift in drag level was driven by concerns of zonal coupling and surface integration errors. It is possible these concerns are unwarranted given the fact that the 81 zone extra-fine grid was built by simply splitting the 17 zone extra-fine grid into smaller pieces. This means the 81 zone extra-fine grid has perfect, point-matched overlap both on the surface and in the volume. The pointmatched overlap was not maintained for the super-fine and ultra-fine grids.

Even though total drag data for GF2 do not fall on a straight line extrapolation of the GF1 fine and extra-fine grid data points, the trend seems reasonable given the level of difficulty involved with this extended grid convergence study. As previously mentioned, obtaining a fully converged solution becomes more challenging with increased grid size. Oscillations in the force and moment convergence histories were present for nearly all of the Pleiades OVERFLOW solutions

with the ultra-fine grid exhibiting the greatest amplitude in drag oscillation at $+/-0.45$ counts. Accounting for this level of error in the ultra-fine drag convergence can help "straighten-out" the GF2 trend line.

Pressure drag is compared in Figure 31 and skin friction drag in Figure 32. It is worth noting that the same scale was used in all three drag plots with a total range of 18 counts. There are no big surprises in the GF1 and GF2 component drag data. The super-fine and ultra-fine grid results fall reasonably close to a linear trend with grid size indicating the data falls within the asymptotic range of grid convergence. The NASA Ames skin friction drag

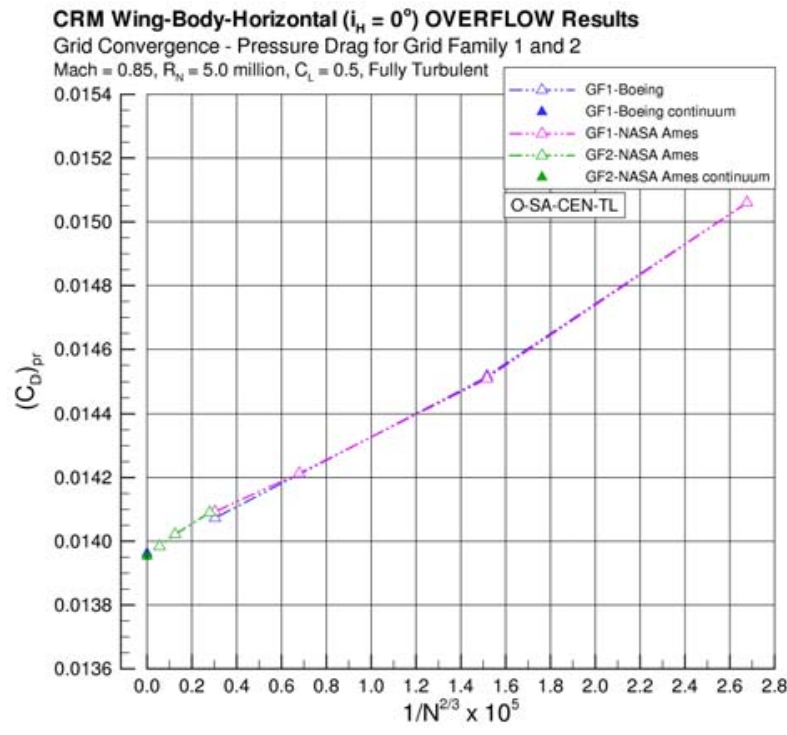

Figure 31. CRM Extended Grid Convergence Study: Pressure Drag.

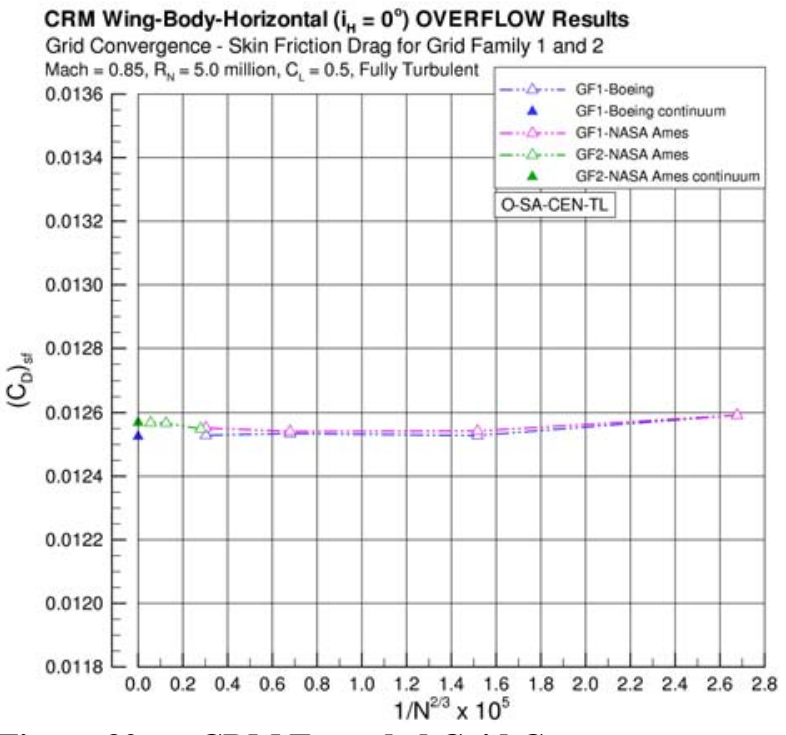

Figure 32. CRM Extended Grid Convergence Study: Skin Friction Drag.

American Institute of Aeronautics and Astronautics 


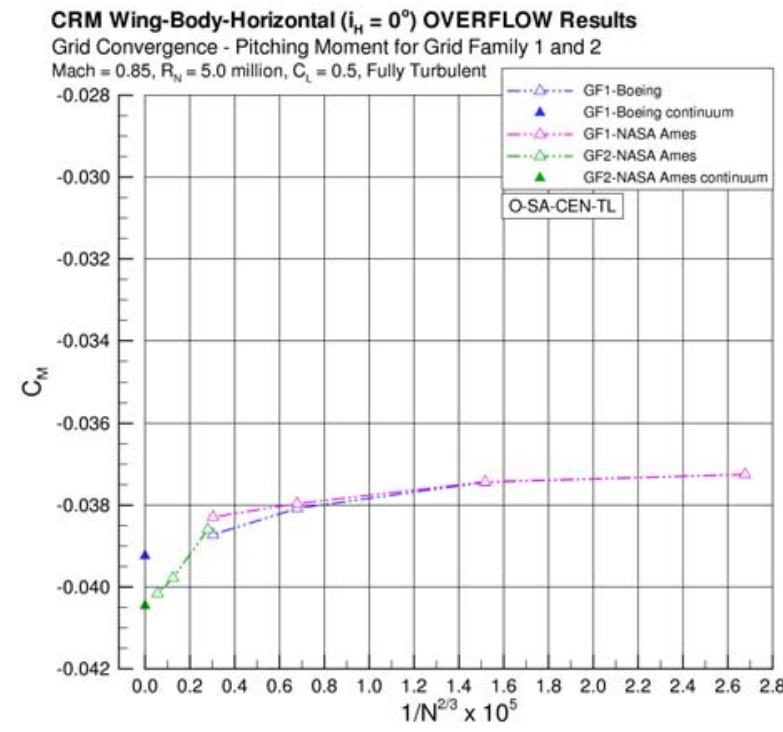

Figure 33. CRM Extended Grid Convergence Study: Pitching Moment.

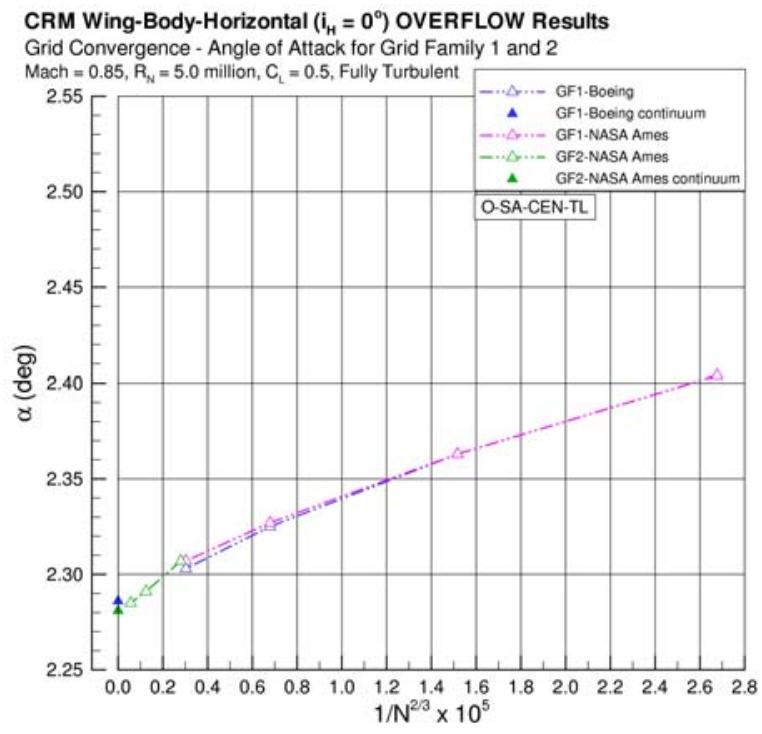

Figure 34. CRM Extended Grid Convergence Study: Angle of Attack.

increases slightly between the fine grid and ultra-fine grid. This is most likely due to a reduction in wing trailingedge separation as observed in the GF1 Boeing results (see Figure 17). With the boundary layer holding-on longer as it approaches the wing trailing-edge, skin friction drag will rise.

The CRM pitching moment results shown in Figure 33 are unlike drag in that the GF2 data represent a clear break from GF1 results. The GF2 extra-fine, super-fine, and ultra-fine pitching moment data form a linear trend in the airplane nose-down direction. The GF1 pitching moment data follow the same trend but with a lower slope. Aft body surface streamlines made from the super-fine solution compare well with the medium grid streamlines shown in Figure 18, so the extended grid refinement does not alter aft body flow separation. In other words, the slope discontinuity between the GF1 and GF2 data is not due to a fundamental change in the flow field near the tail. The discrepancy in pitching moment data will be revisited after summarizing the angle-of-attack results.

Figure 34 shows how the angle-of-attack required to maintain $C_{L}=0.5$ changes with grid size. All three sets of data indicate reduced alphas are needed to hold $\mathrm{C}_{\mathrm{L}}$ as the number of grid points increase. As with pitching moment,

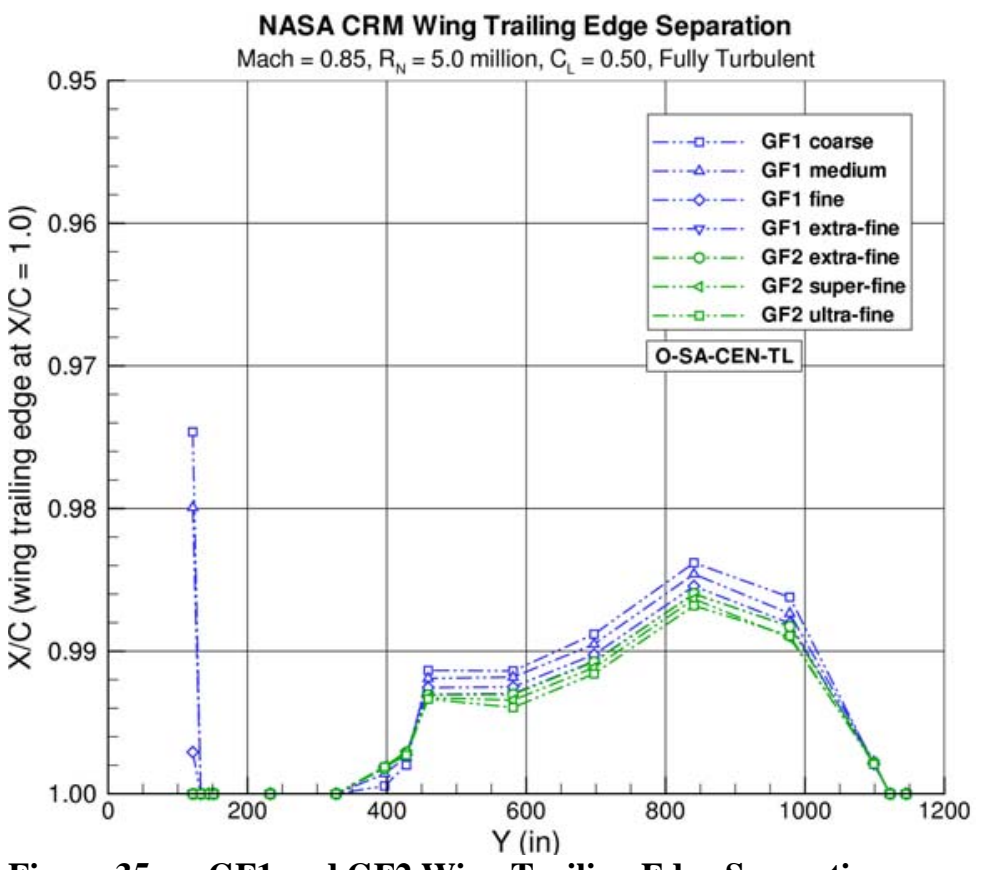

Figure 35. GF1 and GF2 Wing Trailing Edge Separation. there is a slope discontinuity between the NASA Ames GF1 and GF2 data. The data given in Figures 16 and 17 represent one, if not the only, possible explanation for the inverse relationship between angle-ofattack and grid size. To help better understand the GF2 results plotted in Figures 32, 33, and 34, wing trailing edge separation must be revisited.

The amount of separation on the wing's upper surface near the trailing edge is quantified in Figure 35 for the four GF1 grids and three GF2 grids. The plot shows separation at the side-of-body is eliminated with grid refinement as indicated by the data at $\mathrm{Y}=121 \mathrm{in}$. Outboard of the yehudi break, the trend is the same - more grid results in less separation. These findings support the conclusion that skin friction drag grows with grid refinement because of a reduction in separated flow (see Figure 32). Another artifact of a wing with less separation is a reduced angle-of-attack 


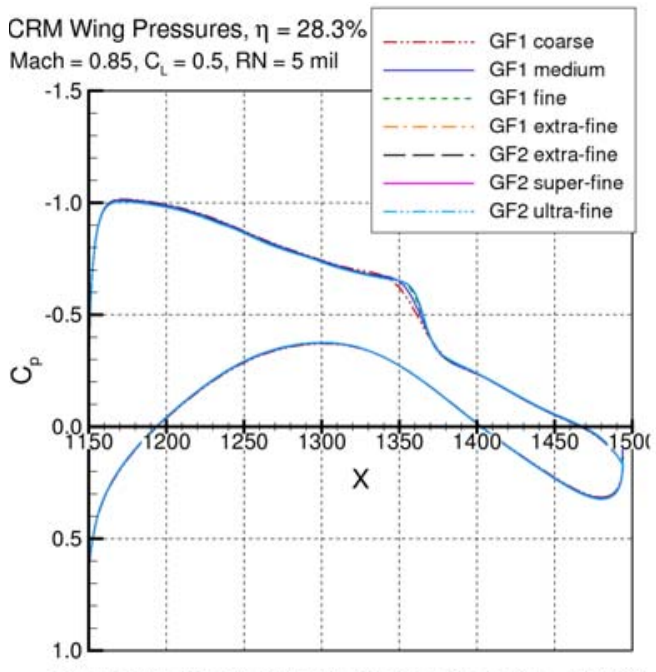

Figure 36a. O-SA-CEN-TL Comparison at $\eta=28.3 \%$
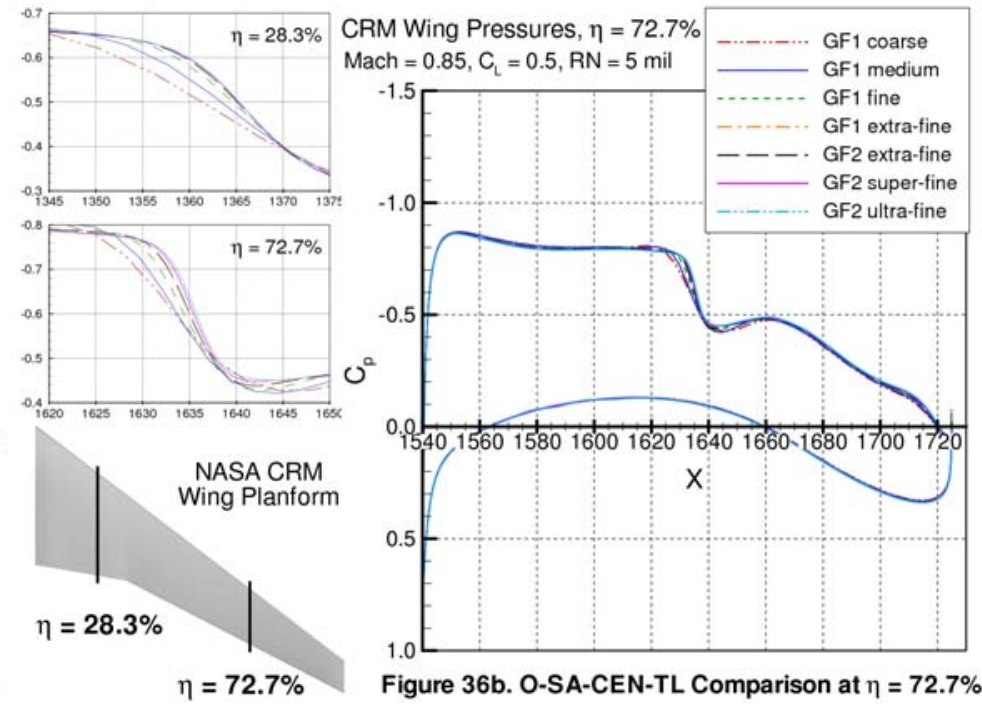

Figure 36. CRM O-SA-CEN-TL Wing Pressure Comparison at $\eta=28.3 \%$ and $72.7 \%$.

for constant lift which, in turn, drives the tail-on pitching moment more negative. While the separation trend given in Figure 35 sheds some light on the direction force, moment, and alpha levels take, it does not help explain the slope discontinuities seen in Figures 33 and 34. Further investigation is needed to explain why the GF2 data follows a different grid convergence line for pitching moment and alpha.

CRM wing pressures are compared at two stations in Figure 36. Figure 36a compares pressure on the inboard wing at a semi-span station of $28.3 \%(\mathrm{Y}=327.074$ in) for all grids analyzed using OVERFLOW. Based on this fullchord comparison, pressure variation is greatest at the shock. The pressures through the shock are easier to see using the smaller plot located in the center of Figure 36 which shows an increase in shock strength with increased grid size. The greatest variation is between the coarse, medium, and fine grid solutions. Some correlation can be made between changes in coarse-medium-fine grid shock strength and breaks in the total drag grid convergence line plotted in Figure 30. There is a very small difference in shock definition between the GF1 fine and extra-fine grids. At this scale, no difference can be seen between the two extra-fine grids or the super-fine and ultra-fine grids. Figure $36 \mathrm{~b}$ compares pressure on the outboard wing at a semi-span station of $72.7 \%$ (Y=840.704in). The full-chord comparison at this station shows a little more variation in upper surface pressures compared to the inboard cut, but the largest effect of grid density is still found at the shock. Again, the smaller plot centered in Figure 36 is used to see how shock definition varies with grid refinement. The same trend is present with a little more difference between the fine and extra-fine grids. Based on these two pressure comparisons, there is no need to go beyond densities found in the extra-fine grid if the goal is a converged shock definition.

\section{Conclusion}

The NASA Common Research Model was analyzed in both wing-body and wing-body-tail configurations using CFL3D and OVERFLOW. This work was initiated for the $4^{\text {th }}$ Drag Prediction Workshop by building a family of structured, overset grids appropriate for use in grid convergence studies. A medium-sized grid consisting of roughly 17 million points for the wing-body-tail configuration was generated first using established guidelines. This grid is considered representative for cruise drag design studies, and all other grids in the family were built from it by systematically removing or adding points. Steps were taken to ensure all grids in the family fall on the same underlying surface definition. The grid family constructed for the workshop consisted of four grids ranging in size from 7.2 million points up to 189.4 million points. Following the workshop, two additional grids were built as an extension of the original four with grid sizes of 714.2 million points and 2.4 billion points.

OVERFLOW results were obtained for all grid densities under study using various combinations of differencing scheme, turbulence model, and extent of viscous term calculation. CFL3D was run for the three coarsest grids as overset-related convergence issues prevented denser grids from being analyzed within a reasonable amount of time. The computed force and moment results were assumed to be second-order accurate so they could be plotted as a function of grid size using Richardson extrapolation. This appears to be a good assumption given most of the drag data form linear trends indicating asymptotic grid convergence was achieved. By extrapolating data to the 
continuum using results from the two finest grids analyzed, CFL3D and OVERFLOW grid converged drag was estimated to be in very good agreement differing by only 1.3 counts. Turbulence model effects were significant with the two-equation SST model predicting 7 counts more drag than the one-equation SA model based on the medium grid OVERFLOW results. The SST solution predicts a stronger wing shock that is swept forward relative to SA, so most of the 7 count drag difference appears to be due to shock drag. The two turbulence models also predict different effects of grid refinement on aft body flow separation leading to a large discrepancy in pitching moment data.

The medium grid density was used to analyze the CRM wing-body and wing-body-tail configurations with multiple tail settings at several angles-of-attack. The force and moment results were post-processed to compute a trimmed drag polar. In general, CFL3D and OVERFLOW are in very good agreement. The medium grid drag level for a trimmed configuration is 273.0 counts for CFL3D and 276.1 counts for OVERFLOW. The two codes appeared to predict opposite pitch breaks with CFL3D pitching moment data indicating a nose-down break while OVERFLOW was nose-up. This behavior was traced to the effect the differencing scheme has on inboard wing separation at high alpha. When both solvers utilize the same scheme, the pitching moment results are consistent. Inboard wing separation at high angle-of-attack was studied further by analyzing a dense patch grid located on the wing upper surface at the side-of-body. The patch grid was intended to improve grid quality by holding $\mathrm{y}^{+}$spacing in two directions at the wing-body corner. The results indicated that increasing grid resolution significantly increased the size of the side-of-body separation bubble. Furthermore, solving the full Navier-Stokes equations yielded a larger bubble size than solving a thin-layer approximation (similar conclusions have been reported for DPW-III overset grid studies). Although it is not known for sure at this time, based on DPW-III studies it is likely that the side-of-body separation bubble on the CRM remains relatively small at high angle-of-attack. If so, this leads to the interesting conclusion that the turbulence model may be missing key physics for the accurate prediction of wingbody corner flows at extreme conditions and/or on certain geometry. When the CRM flow field is adequately resolved at $4^{\circ}$ angle-of-attack, the turbulence model predicts too large a bubble. Furthermore, this study reveals the danger of drawing conclusions from CFD results that have not been adequately grid-resolved in key regions of the flow field: apparently one can obtain reasonable-looking results in the side-of-body region when the grid in that region is under-resolved, and/or when cross-derivative terms are ignored. Further detailed study on this issue is warranted.

The grid convergence study initiated for DPW-IV was expanded by analyzing a 714.2 million point grid and a 2.4 billion point grid. OVERFLOW was used to obtain solutions on these mega-grids at the design condition, and the results were added to the grid convergence plots. The data seem to support the conclusion that asymptotic grid convergence can be achieved using grids in the 10 million to 200 million point range for a wing-body-tail configuration. There appears to be little return on investment for building, analyzing, and post-processing billionpoint grid systems for use in this type of configuration at its design conditions. However, it may be interesting in future work to apply such fine grids to off-design (separated) conditions. Also, this was a valuable exercise conducted for reasons that go beyond the objectives of the CRM study discussed here. For example, by pushing grid size to such an extreme, weaknesses in the overall process (from grid generation to post-processing) can be exposed. The goal is to document all of the lessons learned in future work.

\section{Acknowledgments}

The authors thank The Boeing Company and The National Aeronautics and Space Administration for their support in the Drag Prediction Workshop series. A special thanks goes to Stuart Rogers, Dennis Jespersen, and William Chan at the NASA Ames Research Center for providing a considerable amount of help building, analyzing, and post-processing the 2.4 billion point overset grid. The Boeing authors also thank David Yeh, Mike Epton, and Ben Rider at The Boeing Company for their post-processing help.

\section{References}

${ }^{1}$ Vassberg, J. C., Buning, P. G., and Rumsey, C. L., "Drag Prediction for the DLR-F4 Wing/Body Using OVERFLOW and CFL-3D on an Overset Mesh," AIAA Paper 2002-0840, 40 ${ }^{\text {th }}$ AIAA Aerospace Sciences Meeting and Exhibit, Reno, NV, January 2002.

${ }^{2}$ Vassberg, J. C., DeHaan, M. A., and Sclafani, A. J., "Grid Generation Requirements for Accurate Drag Prediction Based on OVERFLOW Calculations," AIAA Paper 2003-4124, $16^{\text {th }}$ AIAA Computational Fluid Dynamics Conference, Orlando, FL, June 2003.

${ }^{3}$ Sclafani, A. J., Vassberg, J. C., Harrison, N. A., DeHaan, M. A., Rumsey, C. L., Rivers, S. M., Morrison, J. H., "Drag Prediction for the DLR-F6 Wing/Body and DPW Wing Using CFL3D and OVERFLOW on an Overset Mesh," AIAA Paper 2007-0257, 45 ${ }^{\text {th }}$ AIAA Aerospace Sciences Meeting and Exhibit, Reno, NV, January 2007. 
${ }^{4}$ Vassberg, J. C., Tinoco, E. N., Mani, M., Brodersen, O. P., Eisfeld, B., Wahls, R. A., Morrison, J. H., Zickuhr, T., Laflin, K. R., Mavriplis, D. J., "Summary of the Third AIAA CFD Drag Prediction Workshop," AIAA Paper 2007-0260, 45 ${ }^{\text {th }}$ AIAA Aerospace Sciences Meeting and Exhibit, Reno, NV, January 2007.

${ }^{5}$ Vassberg, J. C., DeHaan, M. A., Rivers, S. M., Wahls, R. A., "Development of a Common Research Model for Applied CFD Validation Studies," AIAA Paper 2008-6919, 26 ${ }^{\text {th }}$ AIAA Applied Aerodynamics Conference, Honolulu, HI, August 2008.

${ }^{6}$ Rivers, S. M., Ditberner A., "Experimental Investigations of a Common Research Model for Applied CFD Validation Studies," AIAA Paper 2010-4218, 28 ${ }^{\text {th }}$ AIAA Applied Aerodynamics Conference, Chicago, IL, June 2010.

${ }^{7}$ Vassberg, J. C., Tinoco, E. N., Mani, M., Zickuhr, T., Levy, D., Brodersen, O. P., Eisfeld, B., Wahls, R. A., Morrison, J. H., Mavriplis, D. J., "Summary of the Fourth AIAA CFD Drag Prediction Workshop," AIAA Paper 2010-4547, 28 ${ }^{\text {th }}$ AIAA Applied Aerodynamics Conference, Chicago, IL, June 2010.

${ }^{8} 4^{\text {th }}$ CFD Drag Prediction Workshop, URL: http://aaac.larc.nasa.gov/tsab/cfdlarc/aiaa-dpw/, email: dpw@,cessna.textron.com, June 2009.

${ }^{9}$ Chan, W. M., Gomez III, R. J., Rogers, S. E., Buning, P. G., "Best Practices in Overset Grid Generation," AIAA Paper 2002-3191, 32 ${ }^{\text {nd }}$ AIAA Fluid Dynamics Conference, St. Louis, MI, June 2002.

${ }^{10}$ Gatzke, T. D., LaBozzetta, W. F., Frinfrock, Johnson, J. A., Romer, W. W., "MACGS: A Zonal Grid Generation System for Complex Aero-Propulsion Configurations,” AIAA Paper 1991-2156, June 1991.

${ }^{11}$ Chan, W. M., Rogers, S. E., Pandya, S. A., Kao, D. L., Buning, P. G., Meakin, R. L., Boger, D. A., Nash, S. M., “Chimera Grid Tools User's Manual," URL: http://people.nas.nasa.gov/ wchan/cgt/doc/man.html, Version 2.1, March 2010.

${ }^{12}$ Nash, S. M., Rogers, S. E., "Manual for Scripts," URL: http://rotorcraft.arc.nasa.gov/cfd/CFD4/CGT/scripts.html, January 2008.

${ }^{13}$ Chan, W. M., Chiu, I. T., Buning, P. G., "User's Manual for the HYPGEN Hyperbolic Grid Generator and the HGUI Graphical User Interface,” NASA TM 108791, October 1993

${ }^{14}$ Suhs, N. E., Rogers, S. E., Dietz, W. E., "PEGASUS 5: An Automated Pre-processor for Overset-Grid CFD," AIAA Paper 2002-3186, 32 ${ }^{\text {nd }}$ AIAA Fluid Dynamics Conference, St. Louis, MI, June 2002.

${ }^{15}$ Salas, M. D., "Digital Flight: The Last CFD Aeronautical Grand Challenge," Journal of Scientific Computing, Vol. 28, Nos. 2/3, September 2006.

${ }^{16}$ CFL3D Version 6.5, URL: http://cfl3d.larc.nasa.gov/Cfl3dv6/cfl3dv6.html, 2007.

${ }^{17}$ Krist, S. L., Biedron, R. T., Rumsey, C. L., “CFL3D User's Manual Version 5.0,” NASA TM 1998-208444, NASA, June 1998.

${ }^{18}$ Nichols, R. H., Buning, P. G., "User's Manual for OVERFLOW Version 2.1t," August 2008.

${ }^{19}$ Spalart, P. R., Allmaras, S. R., “A One-Equation Turbulence Model for Aerodynamic Flows,” AIAA Paper 92-0439, January 1992.

${ }^{20}$ Menter, F. R., "Two-Equation Eddy Viscosity Turbulence Models for Engineering Applications,” AIAA Journal, Vol. 32, No. 8, August 1994, pp. 1598-1605.

${ }^{21}$ Mani, M., Rider, B. J., Sclafani, A. J., Winkler, C., Vassberg, J. C., Dorgan, A. J., Cary, A., Tinoco, E. N., "RANS Technology for Transonic Drag Prediction; A Boeing Perspective of the $4^{\text {th }}$ Drag Prediction Workshop," AIAA Paper 2010-4221, $28^{\text {th }}$ Applied Aerodynamics Conference, Chicago, IL, June 2010. 